\title{
48. VARIATIONS IN SEDIMENT PHYSICAL PROPERTIES AND PERMEABILITY OF MUD-VOLCANO DEPOSITS FROM NAPOLI DOME AND ADJACENT MUD VOLCANOES ${ }^{1}$
}

\author{
Achim Kopf, ${ }^{2}$ M. Ben Clennell, ${ }^{3}$ and Angelo Camerlenghi ${ }^{4}$
}

\begin{abstract}
Active mud volcanoes on the Mediterranean Ridge accretionary prism were sampled during Bannock Cruises 88 and 89 and Ocean Drilling Program Leg 160. Permeability tests on undisturbed whole-round samples from Napoli dome (Site 971) using a back-pressured system at effective stresses that ranged from 200 to $700 \mathrm{kPa}$ revealed low hydraulic conductivities of the clayrich, undisturbed "mud breccias," which ranged from $5 \times 10^{-8}$ to $5 \times 10^{-9} \mathrm{~mm} / \mathrm{s}$. In general, conductivities of sediments from the footwall, flank, and crest of Napoli dome dropped to half their value when the load was incrementally increased from 500 up to $700 \mathrm{kPa}$. Pore volume, initially $50 \%-55 \%$, was reduced to around $25 \%$ after multiple incremental loading between the permeability tests. Experiments on remolded mud breccia from Napoli dome (Site 971) and adjacent mud volcanoes using a geotechnical shear box as well as a Vane apparatus revealed peak shear strengths of 200-400 kPa. Plasticity indices also vary significantly for the mud-volcano deposits (i.e., from $25 \%$ to $>40 \%$ ), presumably because of variations in the composition and amount of the clay fraction. The friction angles obtained with shear tests indicate that montmorillonites $\left(\phi^{\prime}\right.$ peak $\left.=10^{\circ}-15^{\circ}\right)$ and fine grained carbonates $\left(\phi^{\prime}\right.$ peak $\left.27^{\circ}-30^{\circ}\right)$ are the two dominant mineral phases in the matrix. By comparison, hemipelagic sediments (calcareous ooze) show plasticity indices of $<20 \%$ and shear strengths of $>600 \mathrm{kPa}$ with friction angles around $30^{\circ}$.

The driving force of the Mediterranean Ridge mud volcanism is believed to relate to regional tectonic compression resulting from collision of the African and Eurasian plates. The properties of the evaporitic overburden, as well as the degree of overpressuring of mud at some depth, are thought to primarily control the activity. Phases of hemipelagic "background" sedimentation, as indicated by interfingering patterns with mud breccia, are accompanied by a build-up of pressure at depth until the subsurficial, clay-rich muds are forced to extrude again. Low permeabilities, together with the grain-size variation (and indirectly viscosity) of the mud breccias, are considered to have a secondary effect on the episodicity in mud-volcanic activity on the Mediterranean Ridge, resulting from blocking vents.
\end{abstract}

\section{INTRODUCTION AND GEOLOGICAL SETTING}

Mud volcanism in subduction zones is a well-known phenomenon, described from the Barbados accretionary wedge (Brown and Westbrook, 1988; Henry et al., 1990), Makran (Fowler et al., 1985; Barber et al., 1986), Sumba (Breen et al., 1986), and Costa Rica (Shipley et al., 1990). The mechanisms of mud volcanoes formation are strongly dependent on the consolidation and saturation conditions of fine-grained sediments at depth. Brown (1990) proposed a model of the evolution of such sediments' state of consolidation, following a specific stress path that eventually leads to the formation of mud volcanoes and sedimentary diatremes, after gas expansion in the pore spaces. The model was merely theoretical and based on the observation of the distribution of the scaly fabric on exposed fossil mud diapirs. An attempt made by Camerlenghi et al. (1995) to evaluate the consolidation path of mud-volcano sediments was based on surface mud-volcano sediments, lacking the essential control of the sediments' characteristics with depth.

The collection and analysis of new data from boreholes in mud volcanoes is therefore essential to further development of the models that explain the mechanisms and time scale of mud volcanic activity. In particular, an explanation is needed for the unlithified state of the mud in spite of the considerable burial depth within or beneath the accretionary prism (e.g., Schulz et al., 1997).

${ }^{1}$ Robertson, A.H.F., Emeis, K.-C., Richter, C., and Camerlenghi, A. (Eds.), 1998. Proc. ODP, Sci. Results, 160: College Station, TX (Ocean Drilling Program).

${ }^{2}$ Geologisches Institut, Albert-Ludwigs-Universität Freiburg, Albertstrasse 23B, 79104 Freiburg, Federal Republic of Germany. (Present address: GEOMAR, Wischofstrasse 1-3, 24148 Kiel, Federal Republic of Germany.) akopf@geomar.de dom.

${ }^{3}$ Department of Earth Sciences, University of Leeds, Leeds LS2 9JT, United King-

${ }^{4}$ Osservatorio Geofisico Sperimentale, P.O. Box 2011, 34016 Trieste, Italy.
Mud volcanism and mud diapirism on the Mediterranean Ridge accretionary complex were discovered in the eighties (Cita et al., 1981 and 1989; Camerlenghi et al., 1992) and related to the compressional tectonics of the Mediterranean Ridge accretionary complex (e.g., Camerlenghi et al., 1995). Geophysical data recently collected suggest that mud volcanoes are widespread on most of the Mediterranean Ridge and that individual mud domes vary greatly in scale and size (e.g., Cronin et al., 1997; see Fig. 1).

Two contrasting mud domes on the northern margin of the Mediterranean Ridge accretionary complex were drilled during Ocean Drilling Program (ODP) Leg 160 (Emeis, Robertson, Richter, et al., 1996). At both the apparently dormant Milano dome (Site 970; Fig. 1) and the currently active Napoli dome (Site 971; Fig. 1), a transect of holes was drilled from the adjacent clayey silts and hemipelagic oozes, across the flanks, to the crestal area of the structures (Fig. 2). The dominant sediment types are variably clast-rich, matrix-supported muds characterized by very poor sorting with interbedded and interfingering hemipelagic sediments (Fig. 3; Robertson et al., 1996; Flecker and Kopf, 1996). The layered nature of the sediments and the vertical and lateral gradational changes from "mud breccia" to hemipelagic oozes, associated with mud-debris flows, strongly suggests their extrusive origin. Biostratigraphic ages of the interbedded hemipelagic deposits indicate that mud volcanism at Milano dates from at least 1.5-1.75 Ma, whereas the Napoli mud volcano was initiated more than 1.25-1.5 Ma (Fig. 2).

Geochemical studies show evidence of upward flow of methane and higher hydrocarbon gases and also suggest the presence of gas hydrates in the crestal areas of both the Milano and Napoli mud volcanoes (see Emeis, Robertson, Richter, et al., 1996). Clasts within the mud breccia are predominantly calcilutites, pelagic carbonates (Burdigalian-Langhian age and younger), litharenites, and, to a lesser extent, solid halite of postulated Messinian age. However, a small quan- 


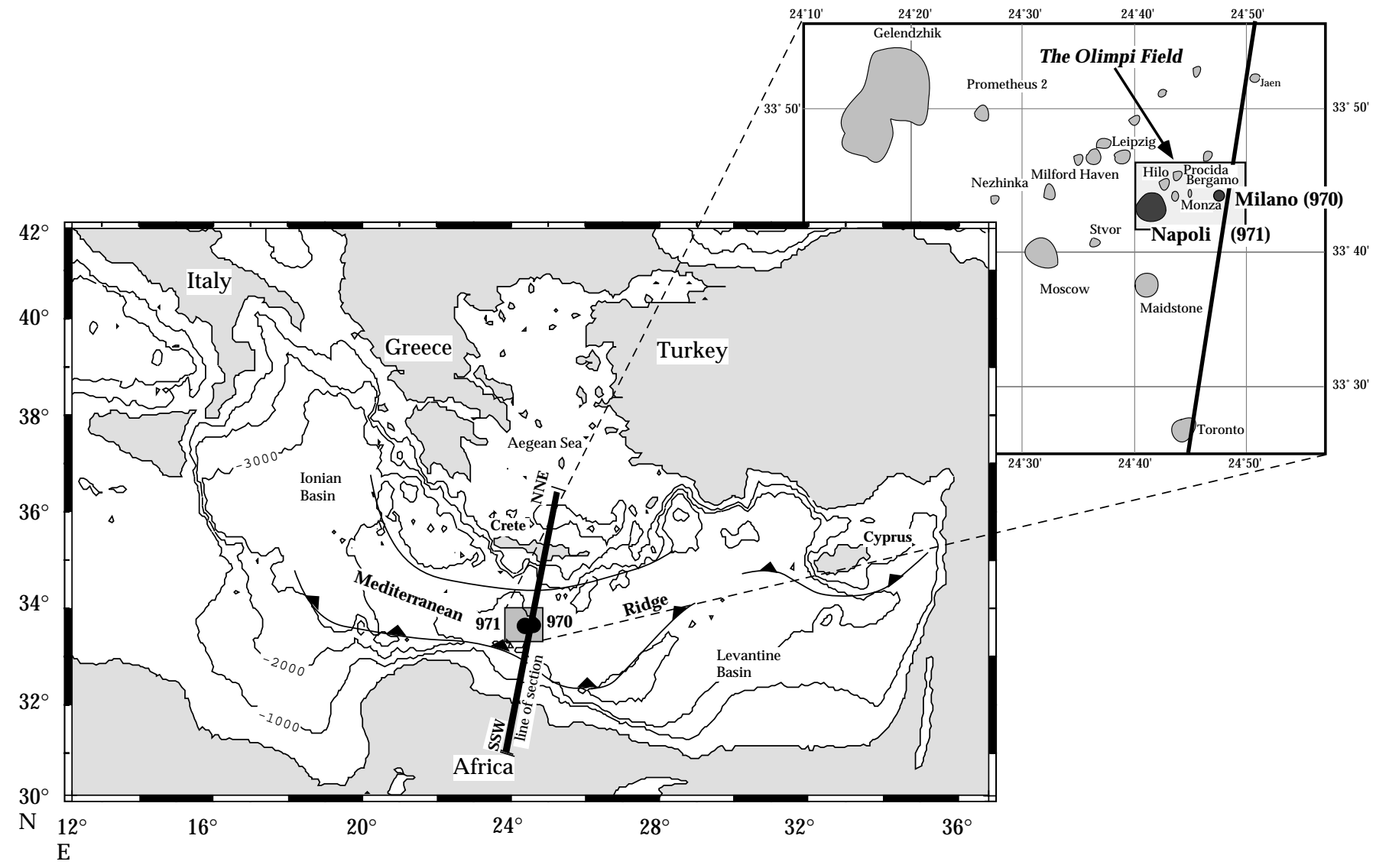

Figure 1. Outline map of the Eastern Mediterranean showing the location of the Milano mud volcano (Site 970) and Napoli mud volcano (Site 971) on the Mediterranean Ridge, which was drilled during Leg 160 (Emeis, Robertson, Richter, et al., 1996). The roughly north-south-oriented line across the accretionary wedge is where the sketch of the cross section (Fig. 9) is placed. The enlarged area shows the Olimpi mud-volcano field (after Kopf et al., in press).

tity of fibrous calcite (thought to have precipitated from methanederived fluid expulsion; e.g., Kopf et al., 1995) was also found. The early Miocene age of some of the clasts suggests that the source of unconsolidated muds is presumed to be somewhere beneath a layer of Messinian evaporites.

During cruises of the Bannock in 1988 and 1989, gravity cores were recovered from Napoli, Monza, and Hilo mud domes (for location see Fig. 1, upper right corner; Cita et al., 1989). On the Napoli and Monza volcanoes, typical mud breccias are overlain by a thin hemipelagic sediment cover both at the top and on several slope sites. In addition, in a basin beside Napoli dome, the transition from mudvolcano deposits to hemipelagic sediment exists (transitional sediments are referred to as mixed sediment). From the crest of the Hilo mud dome, layered successions of nannofossil ooze and sapropels were recovered. None of the cores exceeds $5 \mathrm{~m}$ in length.

\section{Purpose of the Study and Previous Work}

In order to understand the mechanisms and timing of mud volcanic activity in relation to the tectonic evolution of the Mediterranean Ridge, different geotechnical approaches were used. This seemed particularly important as an explanation is needed for the unlithified state of the mud, although there is evidence for considerable burial depth for several million years within or beneath the accretionary prism (e.g., Schulz et al., 1997).

Previous investigators evaluated sediment physical properties (i.e., water content, density), shear strength, and permeability of soil and sediment (Casagrande, 1936, 1948; Bjerrum, 1954; Skempton,
1964; Boyce, 1976; Lupini et al., 1981; Schultheiss and Gunn, 1985). Testing procedures concerning the behavior of different soil sediment types from engineering sciences (e.g., Casagrande, 1936; 1948; Bjerrum, 1954; Skempton, 1964) were applied to fine-grained marine sediments (e.g., Boyce, 1976; Lee, 1985). The residual strength of soils has been studied applying different testing procedures, and the relationships between physical properties of deep marine sediments and their mineralogy, pore fluid chemistry, and parameters like lithostatic, hydrostatic, and pore fluid pressure as well as temperature have been shown. Much of this work has been summarized by Lupini et al. (1981) and Moore (1991), and therefore will not be repeated here. The permeability of soils and rocks is of growing importance in engineering geosciences. The development of laboratory measurement methods as well as characteristics of different materials have been reviewed and outlined by Tavenas et al. (1983a, 1983b). In an extensive study, permeability was determined for several types of marine sediments (Bryant et al., 1975; Schultheiss and Gunn, 1985; Taylor and Leonard, 1990).

In this paper we present the geotechnical characterization of mudvolcano sediments from the analysis of five samples taken during previous cruises of the Bannock in 1988 and 1989 (e.g., Cita and Camerlenghi, 1990) as well as on five whole-round samples from Leg 160 (Emeis, Robertson, Richter, et al., 1996) down to 200 meters below seafloor (mbsf). Studying the range of plastic response, shear resistance, and permeability of the mud can be a key to explain why there is unlithified material at depth for a period of several million years. For the first time, a considerable number of undisturbed core samples (down to $200 \mathrm{mbsf}$ ), as well as remolded sediment from mud 


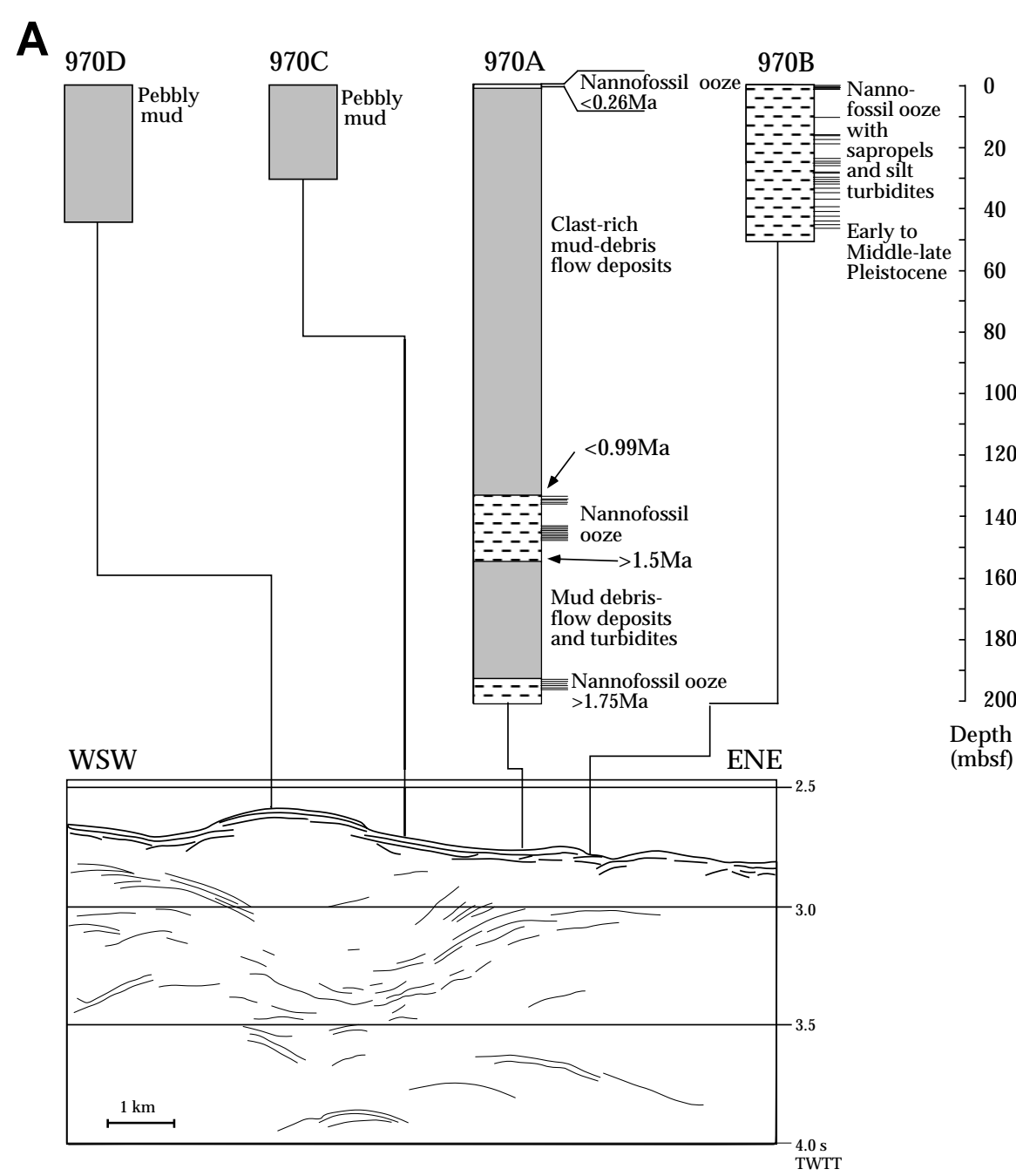

B

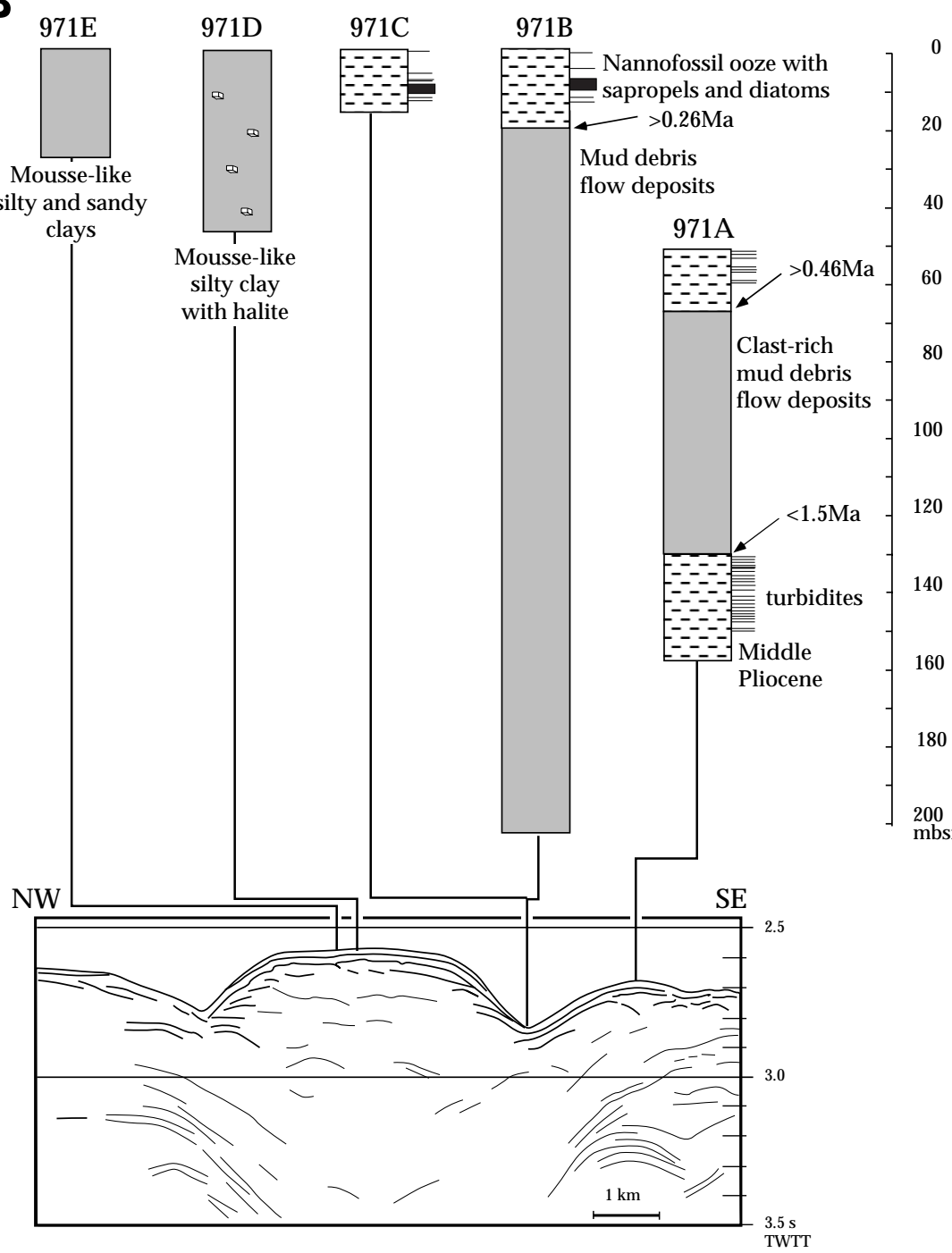

Figure 2. Summary of the lithostratigraphy of the (A) Milano and (B) Napoli mud volcanoes drilled during Leg 160. The most prominent seismic reflectors visible in the migrated time sections are shown at the bottom. Note the presence of inward-dipping reflectors beneath the flanks of both mud domes as well as a depressional moat surrounding Napoli dome (after Robertson et al., 1996) 

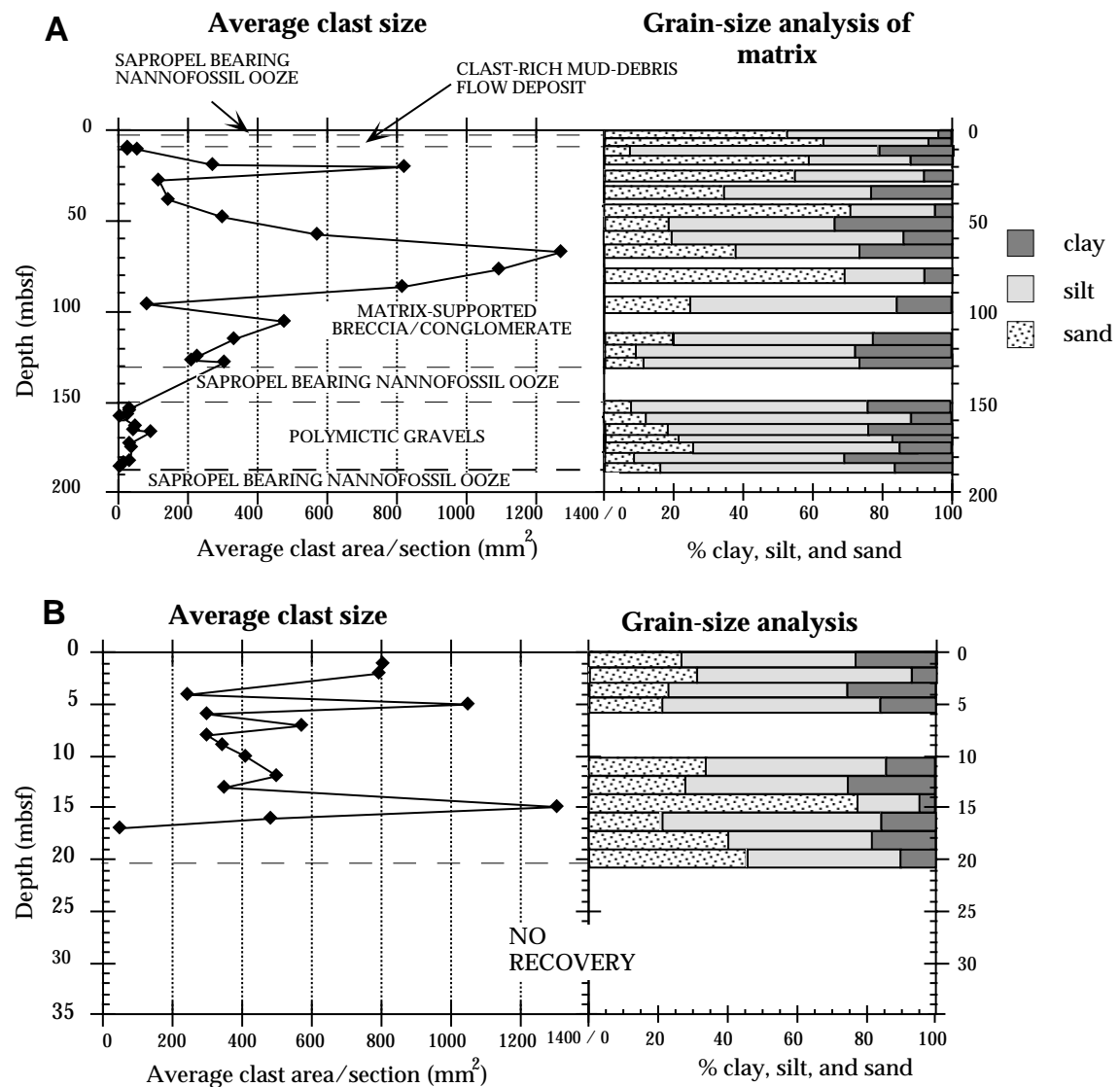

Figure 3. Grain-size and clast-area variation with depth at three holes drilled along a transect into the Milano dome. A. Hole 970A. B. Hole 970C. C. Hole 970D. Data were collected from the different mudvolcano deposits only (after Kopf et al., in press). (Continued next page.)

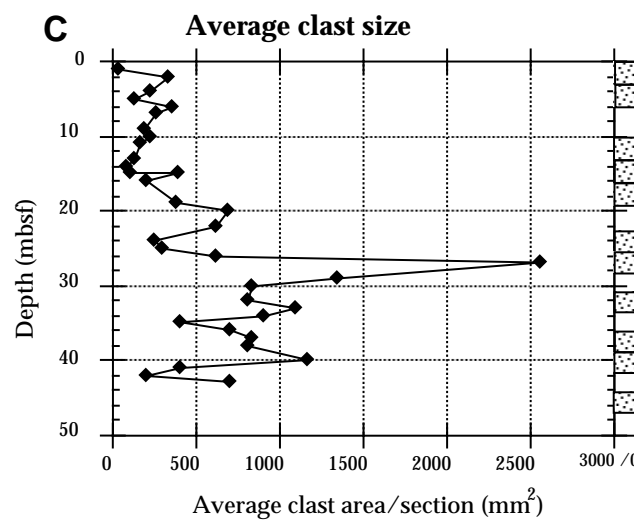

Grain-size analysis

volcanoes, was available. The results will be related to previous mudvolcano studies (e.g., Yassir, 1990; Clennell, 1992; Barber et al., 1986).

\section{LABORATORY TESTING METHODS $\mathrm{X}$-ray Diffraction and Grain-Size Analysis}

Five X-ray diffraction (XRD) determinations of the mineralogical composition of the clay-sized fraction of each whole-round sample were carried out to complement the shipboard and post-cruise XRD analysis of the bulk mud-volcano sediments (Emeis, Robertson, Richter, et al., 1996 and Robertson and Kopf, Chap. 45, this volume, respectively). The XRD analyses were conducted on dried smear slides of the finest fraction $(<2 \mu \mathrm{m})$ obtained by double centrifuging of the sediment sample dispersion.
The grain-size analyses on the matrix of the mud breccia were made using a Coulter LS 100 particle-size analyzer postcruise by Flecker and Kopf (1996). We also examined 14 more samples to cover inadequately sampled intervals, as well as the Leg 160 wholeround samples (Table 1). To more easily compare the samples, we used the same analyzer.

\section{Physical Properties}

Grain density $G_{S}\left(\mathrm{~g} / \mathrm{cm}^{3}\right)$, wet bulk density $\gamma_{B}\left(\mathrm{~g} / \mathrm{cm}^{3}\right)$, dry bulk density $\gamma_{D}\left(\mathrm{~g} / \mathrm{cm}^{3}\right)$, water content $W(\mathrm{wt} \%)$, porosity $n$ (vol\%), and void ratio $e$ (dimensionless) for mud-volcano sediments recovered from Leg 160 were determined as part of the shipboard measurements (see "Physical Properties" section, "Explanatory Notes" chapter, in Emeis, Robertson, Richter, et al., 1996). In addition, we calculated: 
D

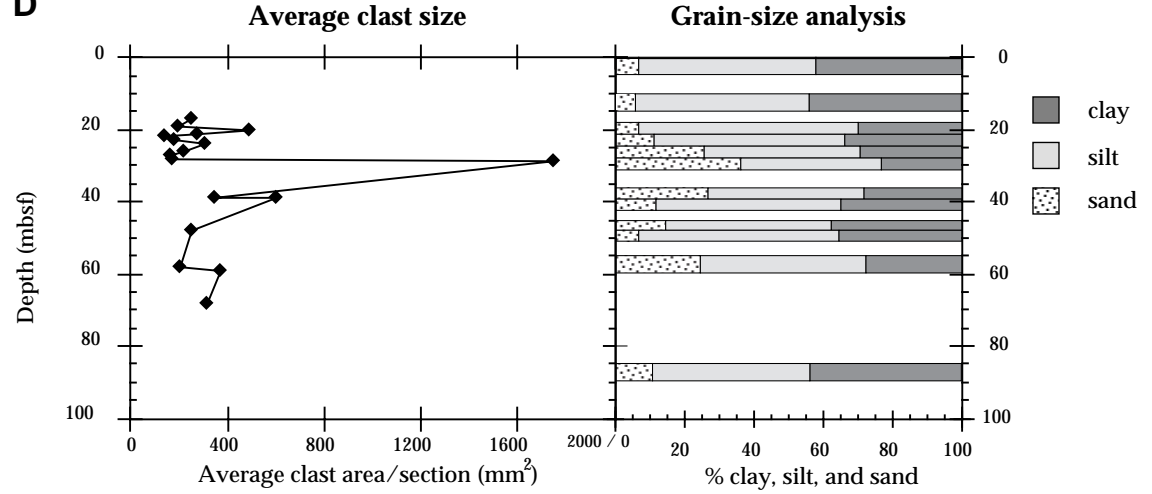

$\mathbf{E}$

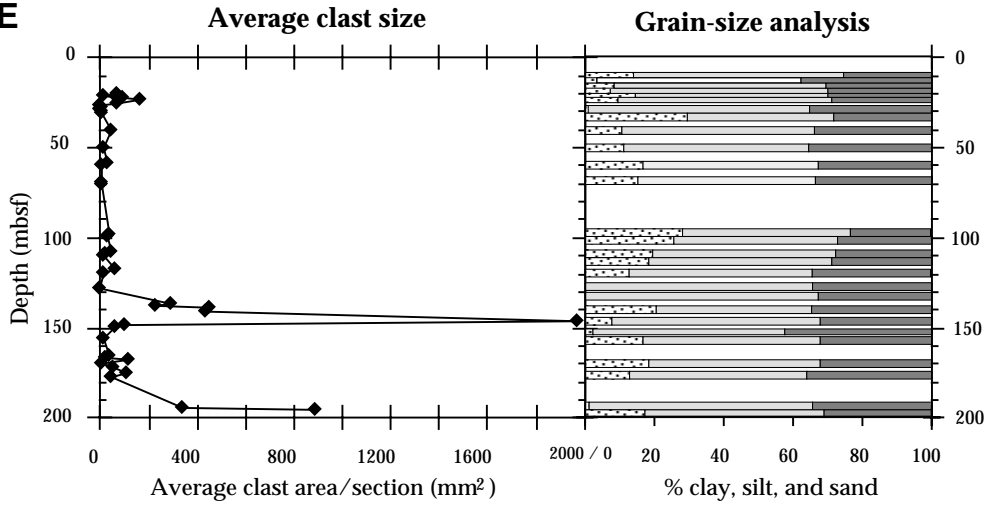

$\mathbf{F}$
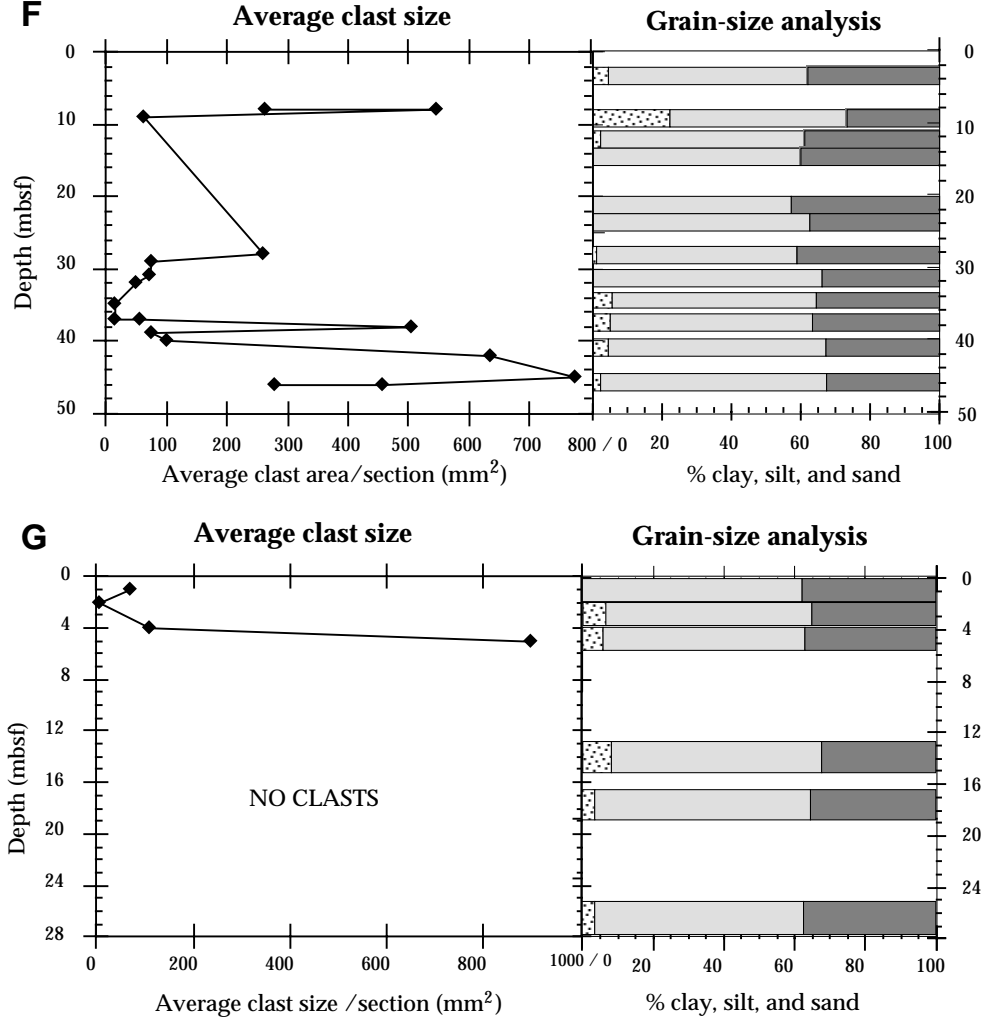

Figure 3 (continued). Grain-size and clast-area variation with depth at three holes drilled along a transect into the Napoli dome. D. Hole 971A. E. Hole 971B. F. Hole 971D. G. Hole 971E. Data were collected from the different mud-volcano deposits only (after Kopf et al., in press).
(A) wet and dry water contents as:

$$
W_{(d r y)}=W_{W} / W_{g} ; \quad W_{(w e t)}=W_{W} / W \quad(\%),
$$

where $W$ is the total wet sample weight, $W_{W}$ is the weight of the water and $W_{g}$ is the dry weight of the sample.

Atterberg limits (Lambe and Whitman, 1969; Head, 1980), and the original water content of the sealed whole-round (WR) samples 
Table 1. Results from shear box tests and grain-size analyses.

\begin{tabular}{|c|c|c|c|c|c|c|c|c|c|c|c|}
\hline \multirow[b]{2}{*}{ Core, section, interval $(\mathrm{cm})$} & \multirow[b]{2}{*}{$\begin{array}{l}\text { Depth } \\
\text { (mbsf) }\end{array}$} & \multicolumn{4}{|c|}{ Shear tests } & \multicolumn{6}{|c|}{ Grain size analyses } \\
\hline & & $\begin{array}{l}\text { Peak strength } \\
(\mathrm{kPa})\end{array}$ & $\begin{array}{l}\phi \mathrm{p} \\
\left({ }^{\circ}\right)\end{array}$ & $\begin{array}{l}\text { Residual strength } \\
(\mathrm{kPa})\end{array}$ & $\begin{array}{l}\phi \mathrm{r} \\
\left(^{\circ}\right)\end{array}$ & $\begin{array}{l}\text { Clay } \\
(\%)\end{array}$ & $\begin{array}{l}\text { Silt } \\
(\%)\end{array}$ & $\begin{array}{c}\text { Sand } \\
(\%)\end{array}$ & $\begin{array}{l}\text { Mean } \\
(\mu \mathrm{m})\end{array}$ & Skewness & $\begin{array}{l}\text { Standard } \\
\text { deviation }\end{array}$ \\
\hline $\begin{array}{l}\text { Hemipelagic sediment } \\
\text { Nannofossil ooze: }\end{array}$ & 2535 & 621 & 20 & 580 & 3 & 2325 & 7655 & & 2010 & 21 & 4042 \\
\hline $\begin{array}{l}\text { Sapropel: } \\
\quad \text { 160-971A-10X-4, 140-150 }\end{array}$ & 25.45 & 253 & 11.5 & $<226$ & $<10.3$ & 10.68 & 85.22 & 4.1 & 75.39 & 3.25 & 155.4 \\
\hline $\begin{array}{l}\text { Transitional sediment } \\
160-971 \mathrm{~A}-4 \mathrm{H}-2,132-150\end{array}$ & 25.32 & 376 & 28 & 211 & 30.1 & 21.04 & 66.96 & 12 & 25.28 & 2.4 & 42.63 \\
\hline $\begin{array}{l}\text { Mud-volcano sediment } \\
160-971 \mathrm{~B}-16 \mathrm{X}-3,99-119\end{array}$ & 139.93 & $\begin{array}{l}199 \\
251\end{array}$ & $\begin{array}{l}29.3 \\
17.9\end{array}$ & $\begin{array}{l}338 \\
155\end{array}$ & $\begin{array}{l}17.7 \\
112\end{array}$ & 38.83 & 61.17 & - & 6.52 & 2.38 & 8.36 \\
\hline $\begin{array}{l}\text { 160-971D-3H-1, 130-150 } \\
160-971 \mathrm{~B}-19 \mathrm{X}-1,130-150\end{array}$ & $\begin{array}{r}18.8 \\
166.2\end{array}$ & $\begin{array}{l}348 \\
399 \\
295\end{array}$ & $\begin{array}{l}27.7 \\
30.4 \\
23.4\end{array}$ & $\begin{array}{l}273 \\
260 \\
282\end{array}$ & $\begin{array}{l}21.8 \\
20.9 \\
22.5\end{array}$ & $\begin{array}{l}22 \\
18.63\end{array}$ & $\begin{array}{l}69.54 \\
77.2\end{array}$ & $\begin{array}{l}8.46 \\
4.17\end{array}$ & $\begin{array}{l}19.99 \\
18.7\end{array}$ & $\begin{array}{l}2.61 \\
1.77\end{array}$ & $\begin{array}{l}31.9 \\
21.63\end{array}$ \\
\hline
\end{tabular}

were determined postcruise in the Department of Earth Sciences of the University of Leeds. The plasticity index $I_{p}(\%)$ has been calculated as difference between the liquid $\left(W_{L}\right)$ and plastic $\left(W_{P}\right)$ limits. All physical properties of the samples taken during previous Bannock cruises were measured onshore with an analogous procedure in the geotechnical laboratory of the Department of Earth Sciences of the University of Milano.

\section{Sediment Shear Strength}

Undrained shear strength $\left(\mathrm{S}_{\mathrm{U}}\right)$ was determined on split cores during Leg 160 (Emeis, Robertson, Richter, et al., 1996). In addition to these determinations, we applied the Wykeham-Farrance motorized vane shear device (following the procedures of Boyce [1976]), to determine both peak $\left(\mathrm{S}_{\mathrm{P}}\right)$ and residual shear strength $\left(\mathrm{S}_{\mathrm{R}}\right)$. A WykehamFarrance standard geotechnical shear box was used for the WR samples from Leg 160 (e.g., ASTM D3080-90, 1991). Samples were remolded (because of for lack of undisturbed material), squeezed into a cell of stainless steel $(\sim 60 \times 60 \times 30 \mathrm{~mm}$ inner size $)$, and then loaded initially with $1 \mathrm{~kg}$ (equivalent to $\sim 44 \mathrm{kPa}$ ). Thereafter, the sample was consolidated with a loading increment ratio of one (following Demars and Chaney, 1992 [ASTM STP 777]; BS1377, 1991) until it reached $\sim 1100 \mathrm{kPa}$. This sample, which was assumed to be normally consolidated, was then sheared at a rate of $0.002 \mathrm{~mm} / \mathrm{min}$ for four days to ensure that failure occurred. The samples were slightly overconsolidated by loading them to an equivalent of $2300 \mathrm{kPa}$ and then unloading them to half the load (importing an overconsolidation ratio of 2 on the sediment) before shearing. We used these tests to assess the degree of hardening and brittleness induced by overconsolidation.

After testing, samples were air-dried and divided into chips for thin section preparation and to make small subsamples for scanning electron microscope (SEM) study.

\section{Oedometer Tests}

Non-back-pressured consolidation tests were conducted on five sediment specimens (height $=2 \mathrm{~cm}$, diameter $=5 \mathrm{~cm}$ ) from Bannock cruises. From each sample, three specimens have been tested for consolidation, one trimmed in vertical direction, one trimmed in horizontal direction, and one remolded at the natural water content. In order to avoid complications caused osmotic effects between the saline pore water and the distilled water of the consolidation apparatus, cells were filled with artificial seawater to simulate in situ conditions $(\mathrm{Ca}-$ merlenghi, 1991). Consolidation was attained for load increments be- tween 12.5 and $2400 \mathrm{kPa}$ vertical effective stress (the increments are outlined in Table 2). Readings were taken manually after having allowed equilibration for $24 \mathrm{hr}$ after each loading increment. The following parameters were calculated from the results of these tests:

(B) the coefficient of consolidation $c_{V}$, which dictates the time rate of volume and pore pressure change in the sediment when loaded:

$$
c_{V}=T_{V(50)} H_{(50)}^{2} / t_{(50)} \quad\left(\mathrm{cm}^{2} / \mathrm{s}\right),
$$

where $T_{V(50)}=0.197$ (i.e., a time factor when $50 \%$ consolidation has taken place), $H_{(50)}=H / 2-d H(H=$ original sample thickness, $d H=$ thickness when $50 \%$ consolidation has taken place), and $t_{(50)}$ is the time when $50 \%$ consolidation has taken place;

(C) the coefficient of volume compressibility, $m_{V}$, for one-dimensional loading

$$
m_{V}=d h /(h d P) \quad\left(\mathrm{cm}^{2} / \mathrm{kg}\right),
$$

where $h$ is the initial thickness, $d h$ its variation with applied pressure, and $d P$ the pressure variation (i.e., change in effective stress);

(D) the theoretical hydraulic conductivity $K$, deduced from the one-dimensional consolidation test using Terzaghi's theory (Lambe and Whitman 1979):

$$
K=c_{V} m_{V} \gamma_{W} \quad(\mathrm{~cm} / \mathrm{s}),
$$

where $c_{V}$ is the coefficient of consolidation, $m_{V}$ is the coefficient of volume compressibility, and $\gamma_{W}$ is the unit weight of artificial ocean water. The hydraulic conductivity is a critical parameter which controls the rate of fluid flow in the sediments, and is directly related to $c_{V}$ and the intrinsic permeability as shown above and below;

(E) the void ratio $e$ as:

$$
e=\left(G s \gamma_{w} h A s / W s\right)-1 \quad \text { (dimensionless) }
$$

where $G s$ is the grain density $(\mathrm{g} / \mathrm{cm} 3), \gamma_{w}$ is the water specific gravity (dimensionless), $h$ is the specimen height $(\mathrm{cm}), A s$ is the specimen area $\left(\mathrm{cm}^{2}\right)$, and $W s$ the weight of the solid $(\mathrm{g})$;

(F) the porosity $n$ as: 
Table 2. Results from oedometer and permeability tests.

\begin{tabular}{|c|c|c|c|c|c|c|c|c|c|c|}
\hline \multirow[b]{2}{*}{ Core, section, interval $(\mathrm{cm})$} & \multicolumn{6}{|c|}{ Oedometer tests } & \multicolumn{4}{|c|}{ Permeability tests } \\
\hline & $\begin{array}{c}\text { Differential } \\
\text { pressure } \\
(\mathrm{kPa})\end{array}$ & $\begin{array}{l}\text { Void } \\
\text { ratio } \\
(e)\end{array}$ & $\begin{array}{l}\text { Porosity } \\
(\%)\end{array}$ & $\begin{array}{c}k \\
(\mathrm{~mm} / \mathrm{s})\end{array}$ & $\begin{array}{c}m v \\
\left(\mathrm{~cm}^{2} / \mathrm{kg}\right)\end{array}$ & $\begin{array}{c}C v \\
\left(\mathrm{~cm}^{2} / \mathrm{s}\right)\end{array}$ & $\begin{array}{c}\text { Effective } \\
\text { stress } \\
(\mathrm{kPa})\end{array}$ & $\begin{array}{c}\text { Sample } \\
\text { height } \\
(\mathrm{mm})\end{array}$ & $\begin{array}{c}\text { Permeability } \\
(\mathrm{mm} / \mathrm{s})\end{array}$ & $\begin{array}{c}\text { Equilibrium } \\
\text { differential } \\
\text { pressure }(\mathrm{kPa})\end{array}$ \\
\hline \multirow{2}{*}{\multicolumn{11}{|c|}{ Mud-volcano deposits }} \\
\hline & 12.5 & 1.232 & 55.2 & - & - & - & - & - & - & \\
\hline & 25 & 1.195 & 54.4 & $9.02 \mathrm{E}-08$ & 0.14240 & 0.00063000 & - & 二 & 二 & \\
\hline & 37.5 & 1.161 & 53.7 & $2.20 \mathrm{E}-08$ & 0.11151 & 0.00019700 & - & - & - & \\
\hline & 56.2 & 1.121 & 52.9 & $2.13 \mathrm{E}-08$ & 0.072100 & 0.00029600 & - & - & - & \\
\hline & 75 & 1.087 & 52.1 & $1.24 \mathrm{E}-08$ & 0.064900 & 0.00019100 & - & - & - & \\
\hline & 112.5 & 1.03 & 50.7 & $1.58 \mathrm{E}-08$ & 0.066500 & 0.00023800 & - & - & - & \\
\hline & 187.5 & 0.944 & 48.6 & $1.50 \mathrm{E}-08$ & 0.048800 & 0.00030800 & - & - & - & \\
\hline & 337.5 & 0.849 & 45.9 & $9.53 \mathrm{E}-09$ & 0.030700 & 0.00031000 & - & - & - & \\
\hline & 637.5 & 0.744 & 42.7 & $5.53 \mathrm{E}-09$ & 0.017000 & 0.00033600 & - & - & - & \\
\hline & 1237.5 & 0.634 & 38.8 & $2.86 \mathrm{E}-09$ & 0.016500 & 0.00030700 & - & - & - & \\
\hline & 1237.5 & 0.624 & 38.4 & $2.00 \mathrm{~L}-0$ & - & -0.00050 & - & - & - & \\
\hline & 637.5 & 0.634 & 38.8 & - & - & - & - & - & - & \\
\hline & 337.5 & 0.651 & 39.4 & - & - & - & - & - & - & \\
\hline & 187.5 & 0.671 & 40.2 & - & - & - & - & - & - & \\
\hline & 112.5 & 0.689 & 40.8 & - & - & - & - & - & - & \\
\hline & 75 & 0.7 & 41.2 & - & - & - & - & - & - & \\
\hline & 56.2 & 0.708 & 41.5 & - & - & - & - & - & - & \\
\hline & 37.5 & 0.717 & 41.8 & - & - & - & - & - & - & \\
\hline & 37.5 & 0.72 & 41.9 & - & - & - & - & - & - & \\
\hline & 56.2 & 0.719 & 41.8 & - & - & - & - & - & - & \\
\hline & 75 & 0.717 & 41.8 & - & - & - & - & - & - & \\
\hline & 112.5 & 0.712 & 41.6 & - & - & - & - & - & - & \\
\hline & 187.5 & 0.702 & 41.2 & - & - & - & - & - & - & \\
\hline & 337.5 & 0.683 & 40.6 & - & - & - & - & - & - & \\
\hline & 637.5 & 0.656 & 39.6 & - & - & - & - & - & - & \\
\hline & 1237.5 & 0.617 & 38.2 & - & - & - & - & - & - & \\
\hline & 2437.5 & 0.531 & 34.7 & 1.69E-09 & 0.0093000 & 0.00023200 & - & - & - & \\
\hline & 3637.5 & 0.468 & 31.9 & $6.40 \mathrm{E}-10$ & 0.0073000 & 0.00012200 & - & - & - & \\
\hline & 4837.5 & 0.435 & 30.3 & - & - & - & - & - & - & \\
\hline Bannock BAN 89-02 & 12.5 & 1.154 & 53.6 & - & - & - & - & - & - & \\
\hline & 25 & 1.119 & 52.8 & 3.38E-08 & 0.11443 & 0.000296 & - & - & - & \\
\hline & 37.5 & 1.085 & 52 & $2.21 \mathrm{E}-08$ & 0.1071 & 0.000206 & - & - & - & \\
\hline & 56.2 & 1.044 & 51.1 & $1.76 \mathrm{E}-08$ & 0.08843 & 0.000199 & - & - & - & \\
\hline & 75 & 1.007 & 50.2 & $1.34 \mathrm{E}-08$ & 0.08617 & 0.000156 & - & - & - & \\
\hline & 112.5 & 0.95 & 48.7 & $1.20 \mathrm{E}-08$ & 0.06582 & 0.000183 & - & - & - & \\
\hline & 187.5 & 0.875 & 46.7 & $1.02 \mathrm{E}-08$ & 0.0465 & 0.000219 & - & - & - & \\
\hline & 337.5 & 0.781 & 43.8 & $6.67 \mathrm{E}-09$ & 0.02863 & 0.000235 & - & - & - & \\
\hline & 637.5 & 0.691 & 40.9 & $3.21 \mathrm{E}-09$ & 0.01522 & 0.000211 & - & - & - & \\
\hline & 337.5 & 0.701 & 41.2 & - & - & - & - & - & - & \\
\hline & 187.5 & 0.717 & 41.8 & - & - & - & - & - & - & \\
\hline & 75 & 0.746 & 42.7 & - & - & - & - & - & - & \\
\hline & 37.5 & 0.767 & 43.4 & - & - & - & - & - & - & \\
\hline & 75 & 0.762 & 43.2 & - & - & - & - & - & - & \\
\hline & 187.5 & 0.738 & 42.5 & - & - & - & - & - & - & \\
\hline & 337.5 & 0.714 & 41.7 & - & - & - & - & - & - & \\
\hline & 637.5 & 0.671 & 40.2 & - & - & - & - & - & - & \\
\hline & 1237.5 & 0.587 & 37 & $1.50 \mathrm{E}-09$ & 0.00734 & 0.000204 & - & - & - & \\
\hline & 2437.5 & 0.491 & 32.9 & $7.83 \mathrm{E}-10$ & 0.00448 & 0.000175 & - & - & - & \\
\hline Bannock BAN 89-04 & 12.5 & 0.995 & 49.9 & - & - & - & - & - & - & \\
\hline & 25 & 0.974 & 49.3 & $2.75 \mathrm{E}-08$ & 0.07626 & 0.00036 & - & - & - & \\
\hline & 37.5 & 0.954 & 48.8 & $1.54 \mathrm{E}-08$ & 0.07254 & 0.000212 & - & - & - & \\
\hline & 56.2 & 0.927 & 48.1 & $1.46 \mathrm{E}-08$ & 0.0704 & 0.000207 & - & - & - & \\
\hline & 75 & 0.904 & 47.5 & $8.53 \mathrm{E}-09$ & 0.0564 & 0.000151 & - & - & - & \\
\hline & 112.5 & 0.867 & 46.4 & $9.97 \mathrm{E}-09$ & 0.0459 & 0.000217 & - & - & - & \\
\hline & 187.5 & 0.81 & 44.8 & $1.17 \mathrm{E}-08$ & 0.0356 & 0.000328 & - & - & - & \\
\hline & 337.5 & 0.74 & 42.5 & $8.20 \mathrm{E}-09$ & 0.0214 & 0.000383 & - & - & - & \\
\hline & 637.5 & 0.659 & 39.7 & $4.91 \mathrm{E}-09$ & 0.0128 & 0.000385 & - & - & - & \\
\hline & 337.5 & 0.667 & 40 & - & - & - & - & - & - & \\
\hline & 187.5 & 0.678 & 40.4 & - & - & - & - & - & - & \\
\hline & 75 & 0.699 & 41.2 & - & - & - & - & - & - & \\
\hline & 37.5 & 0.713 & 41.6 & - & - & - & - & - & - & \\
\hline & 75 & 0.711 & 41.6 & 二 & 二 & - & - & - & 二 & \\
\hline & 187.5 & 0.696 & 41.1 & - & - & - & - & - & - & \\
\hline & 337.5 & 0.679 & 40.4 & - & - & - & - & - & - & \\
\hline & 637.5 & 0.647 & 39.3 & - & - & - & - & - & - & \\
\hline & 1237.5 & 0.57 & 36.3 & $1.97 \mathrm{E}-09$ & 0.00627 & 0.000314 & - & - & - & \\
\hline & 2437.5 & 0.482 & 32.5 & $9.09 \mathrm{E}-10$ & 0.00379 & 0.00024 & - & - & - & \\
\hline 971B-16X-03, 99-119 & & & & & & & 300 & 55 & 7.61E-08 & 51 \\
\hline & & & & & & & 500 & 47 & 2.13E-08 & 39 \\
\hline & & & & & & & 700 & 43.6 & 3.26E-09 & 59 \\
\hline 971B-19X-01, 130-150 & & & & & & & 200 & 64 & $4.18 \mathrm{E}-08$ & \\
\hline & & & & & & & 300 & 62 & $1.21 \mathrm{E}-08$ & 67 \\
\hline & & & & & & & 500 & 56 & $1.19 \mathrm{E}-08$ & 43 \\
\hline & & & & & & & 700 & 56 & $5.73 \mathrm{E}-09$ & 44.2 \\
\hline 971D-3H-01, 130-150 & & & & & & & 300 & 48.2 & $2.95 \mathrm{E}-08$ & 35 \\
\hline & & & & & & & 500 & 39.5 & $3.18 \mathrm{E}-08$ & 35.9 \\
\hline & & & & & & & 700 & 34.6 & 7.05E-09 & 56.7 \\
\hline
\end{tabular}


Table 2 (continued).

\begin{tabular}{|c|c|c|c|c|c|c|c|c|c|c|}
\hline \multirow[b]{2}{*}{ Core, section, interval $(\mathrm{cm})$} & \multicolumn{6}{|c|}{ Oedometer tests } & \multicolumn{4}{|c|}{ Permeability tests } \\
\hline & $\begin{array}{l}\text { Differential } \\
\text { pressure } \\
(\mathrm{kPa})\end{array}$ & $\begin{array}{l}\text { Void } \\
\text { ratio } \\
(e)\end{array}$ & $\begin{array}{l}\text { Porosity } \\
(\%)\end{array}$ & $\begin{array}{c}k \\
(\mathrm{~mm} / \mathrm{s})\end{array}$ & $\begin{array}{c}m v \\
\left(\mathrm{~cm}^{2} / \mathrm{kg}\right)\end{array}$ & $\begin{array}{c}C v \\
\left(\mathrm{~cm}^{2} / \mathrm{s}\right)\end{array}$ & $\begin{array}{c}\text { Effective } \\
\text { stress } \\
(\mathrm{kPa})\end{array}$ & $\begin{array}{c}\text { Sample } \\
\text { height } \\
(\mathrm{mm})\end{array}$ & $\begin{array}{l}\text { Permeability } \\
(\mathrm{mm} / \mathrm{s})\end{array}$ & $\begin{array}{c}\text { Equilibrium } \\
\text { differential } \\
\text { pressure }(\mathrm{kPa})\end{array}$ \\
\hline \multicolumn{11}{|l|}{ Mixed sediment } \\
\hline Bannock BAN 89-03 & $\begin{array}{c}12.5 \\
25 \\
37.5 \\
56.2 \\
75 \\
112.5 \\
187.5 \\
337.5 \\
637.5 \\
337.5 \\
187.5 \\
75 \\
37.5 \\
75 \\
187.5 \\
337.5 \\
637.5 \\
1237.5 \\
2437.5\end{array}$ & $\begin{array}{l}1.028 \\
1.011 \\
0.992 \\
0.97 \\
0.952 \\
0.918 \\
0.874 \\
0.806 \\
0.721 \\
0.728 \\
0.737 \\
0.756 \\
0.769 \\
0.766 \\
0.75 \\
0.735 \\
0.705 \\
0.623 \\
0.52\end{array}$ & $\begin{array}{l}50.7 \\
50.3 \\
49.8 \\
49.2 \\
48.8 \\
47.9 \\
46.6 \\
44.6 \\
41.9 \\
42.1 \\
42.4 \\
43 \\
43.5 \\
43.4 \\
42.9 \\
42.4 \\
41.4 \\
38.4 \\
34.2\end{array}$ & $\begin{array}{c}-\overline{\text { 3. }} \\
3.45 \mathrm{E}-08 \\
2.86 \mathrm{E}-08 \\
1.75 \mathrm{E}-08 \\
2.40 \mathrm{E}-08 \\
2.52 \mathrm{E}-08 \\
1.94 \mathrm{E}-08 \\
1.37 \mathrm{E}-08 \\
- \\
- \\
- \\
- \\
- \\
- \\
7.14 \mathrm{E}-09 \\
4.25 \mathrm{E}-09\end{array}$ & $\begin{array}{c}-\overline{ } \\
0.04171 \\
0.04862 \\
0.04111 \\
0.02966 \\
0.02799 \\
0.02016 \\
0.01785 \\
0.0115 \\
- \\
- \\
- \\
- \\
- \\
- \\
0.00545 \\
0.00385\end{array}$ & 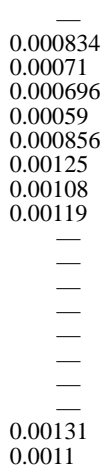 & & & $\begin{array}{l}- \\
- \\
- \\
- \\
- \\
- \\
- \\
- \\
- \\
- \\
-\end{array}$ & \\
\hline $160-971 \mathrm{~A}-4 \mathrm{H}-02,132-150$ & & & & & & & $\begin{array}{r}200 \\
300 \\
500 \\
700 \\
700 \mathrm{r} 1 \\
700 \mathrm{r} 2\end{array}$ & $\begin{array}{l}89 \\
84 \\
53 \\
52 \\
52 \\
52\end{array}$ & $\begin{array}{l}2.93 \mathrm{E}-08 \\
1.77 \mathrm{E}-08 \\
2.46 \mathrm{E}-08 \\
1.31 \mathrm{E}-08 \\
1.39 \mathrm{E}-08 \\
1.55 \mathrm{E}-08\end{array}$ & $\begin{array}{l}65 \\
36.5 \\
43.8 \\
40.2 \\
38 \\
68\end{array}$ \\
\hline $\begin{array}{l}\text { Hemipelagic sediments } \\
\text { Bannock BAN 89-08 }\end{array}$ & $\begin{array}{c}12.5 \\
25 \\
37.5 \\
56.2 \\
75 \\
112.5 \\
187.5 \\
337.5 \\
637.5 \\
337.5 \\
187.5 \\
75 \\
37.5 \\
75 \\
187.5 \\
337.5 \\
637.5 \\
1237.5 \\
2437.5\end{array}$ & $\begin{array}{l}1.221 \\
1.194 \\
1.166 \\
1.14 \\
1.121 \\
1.087 \\
1.044 \\
0.979 \\
0.904 \\
0.91 \\
0.908 \\
0.935 \\
0.949 \\
0.945 \\
0.93 \\
0.916 \\
0.889 \\
0.805 \\
0.678\end{array}$ & $\begin{array}{l}55 \\
54.4 \\
53.8 \\
53.3 \\
52.9 \\
52.1 \\
51.1 \\
49.5 \\
47.5 \\
47.6 \\
47.6 \\
48.3 \\
48.7 \\
48.6 \\
48.2 \\
47.8 \\
47.1 \\
44.6 \\
40.4\end{array}$ & $\begin{array}{c}\text { 9.40E-08 } \\
8.51 \mathrm{E}-08 \\
7.38 \mathrm{E}-08 \\
1.51 \mathrm{E}-08 \\
6.31 \mathrm{E}-08 \\
4.89 \mathrm{E}-08 \\
5.83 \mathrm{E}-08 \\
3.33 \mathrm{E}-08 \\
- \\
- \\
- \\
- \\
- \\
- \\
2.40 \mathrm{E}-08 \\
1.63 \mathrm{E}-08\end{array}$ & $\begin{array}{c}-\overline{ } \\
0.0775 \\
0.07192 \\
0.03788 \\
0.03729 \\
0.02537 \\
0.01252 \\
0.01622 \\
0.00759 \\
- \\
- \\
- \\
- \\
- \\
- \\
0.00415 \\
0.00378\end{array}$ & $\begin{array}{l}\quad \overline{0}-00121 \\
0.00118 \\
0.00195 \\
0.000406 \\
0.000249 \\
0.0039 \\
0.0036 \\
0.00439 \\
\quad- \\
\quad- \\
\quad- \\
\quad- \\
\quad- \\
- \\
0.00579 \\
0.0043\end{array}$ & & & $\begin{array}{l}- \\
- \\
- \\
z \\
z \\
- \\
- \\
- \\
- \\
- \\
-\end{array}$ & \\
\hline
\end{tabular}

$n=100 e /(e+1) \quad(\%)$.

(6)

\section{Permeability Tests}

Permeability, $k$, was directly determined as velocity of the migration of a fluid phase through a sediment of known flow rate (i.e., volume per time unit, $Q$; cross sectional area, $A$; and hydraulic gradient, i) following Darcy's Law

$$
K=Q / A i \quad(\mathrm{~cm} / \mathrm{s})
$$

The intrinsic permeability, $k$, is related to the hydraulic conductivity, $\mathrm{K}$, by adjusting for the unit weight and viscosity $(\eta)$ of the pore water:

$$
k=K \eta / \gamma_{\mathrm{w}} \quad\left(\mathrm{cm}^{2}\right) .
$$

Before each test series, the samples were trimmed to a diameter of $5-6 \mathrm{~cm}$ in order to remove all artificial drill mud along the core liner. Consolidation was achieved using a hydraulically pressurized Wykeham-Farrance permeability cell. Loads were applied in steps of 200 (for some samples), 300,500 , and $700 \mathrm{kPa}$ effective stress $\left(\sigma^{\prime}\right)$, each time waiting for full equilibration of the specimen, as a dissipation of all excess pore pressure dissipated, following ASTM D5084 (ASTM, 1990). The effective stress resulted from the hydraulic pressure in the cell minus the backpressure supplied by a GDS dynamic pore-pressure controller, which monitors both in- and outflow volume to $1 \mathrm{cu}-$ bic $\mathrm{mm}$ and allows all volume changes occurring to be recorded precisely. Specimen drainage was allowed via thin porous disks at either end of the whole-round samples.

After each loading interval, consolidation (uniaxial shortening) was measured by using a displacement transducer on top of the sample. A constant rate of volumetric flow was then induced through the sample with a Harvard Apparatus syringe pump. Infusion or withdrawal rates of 0.1 to $3 \mu \mathrm{L} / \mathrm{min}$ were used. The induced differential pressure across the sample (in no case exceeding $10 \%$ of the effective stress) was recorded by a transducer (Validyne model DP215) with accuracy of $0.5 \mathrm{kPa}$. Inflow and outflow were recorded automatically throughout the experiments by computer. The temperature was held constant at $22 \pm 1^{\circ} \mathrm{C}$ to minimize volume changes caused by differential expansion in clay, viscosity and density changes in the fluid (Schultheiss and Dunn, 1985), and thermal expansion in the upstream end of the system. De-aired water was flushed through the entire sys- 
tem to prevent air entrapment, and was also used for the tests. The effects of not having used saline pore water to protect the equipment from corrosion is discussed below (see also Olson and Daniel, 1981).

\section{EXPERIMENTAL RESULTS}

\section{Mud Breccia Composition and Grain-Size Variation}

Bulk sediment XRD analyses in Emeis, Robertson, Richter, et al. (1996) and Robertson and Kopf (Chap. 45, this volume) have shown that calcite and quartz are the most abundant minerals in most of the samples. The clay fraction contains clay minerals; most abundant are kaolinite and smectite and fine-grained biogenic calcite. Postcruise XRD determinations on the finest fraction of the matrix of mud-debris flow deposits, however, indicate the presence of considerable amounts of chlorite and smectite rather than kaolinite. Clay minerals are more abundant in the crestal holes and in the upper part of the succession of the deep holes.

The compilation of grain and clast size variation shown as stack histograms in Fig. 3 indicate in the majority of the cases a rough fining-upward sequence in each hole. The matrix of the mud-volcano deposits is composed mainly of sand and silt, with a minor clay fraction ranging between $5 \%$ and $40 \%$. None of the samples analyzed contained particles coarser than sand size.

\section{Physical Properties}

Results from shipboard measurements and on WR samples taken during Leg 160 are shown in Figure 4 and Tables 3, 4. The water content at the sediment surface varies generally between $30 \%$ and $45 \%$, and evidently decreases with depth in the hemipelagic sediments, which retain a higher water content than the mud-volcano sediments throughout. Mud-volcano sediments instead show a rather uniform distribution of the water content with depth. The surface porosity ranges from $50 \%$ to $70 \%$, and it is occasionally as low as $40 \%$ in mud-volcano sediment intervals that are rich in clasts (Emeis, Robertson, Richter, et al., 1996). Bulk density is relatively constant when mud-volcano deposits were homogeneous, but increased in intervals with abundant clasts (e.g., the clast-rich, matrix-supported muddebris flow deposits; Fig. 3, e.g., Cores 160-970A-6X through 21X). The grain density of the mud breccia ranges between 2.65 and $2.8 \mathrm{~g} /$ $\mathrm{cm}^{3}$, reflecting the high clay content; thus, hemipelagic deposit densities are found to be $2.75-3.2 \mathrm{~g} / \mathrm{cm}^{3}$ because of their carbonate content (Tables 3, 4).

\section{Atterberg Limits}

The variation of liquid limits $\left(W_{L}\right)$ and plastic limits $\left(W_{P}\right)$ is illustrated in Figure 5. The plasticity indices vary between mud-volcano deposits from the flank $(24 \%-30 \%)$ to the crest $(42 \%)$, whereas the transitional and hemipelagic sediment reaches only $19 \%-27 \%$ and $18 \%$ respectively (Table 5). Notably, the clay-rich sediment from the crest of Napoli dome (Fig. 3) shows the broadest plasticity range (Fig. 5). The higher plasticity of the mud-volcano deposits results from higher contents of swelling clays and lower permeabilities.

The original water contents of all mud-volcano deposits tested lie in between $W_{L}$ and $W_{P}$. The transitional whole-round sample has its natural water content close to the plastic limit (i.e., brittle behavior). By contrast, the natural water content and the liquid limit of the nannofossil ooze are very similar, indicating that this material may behave like a fluid if put under undrained stress conditions.
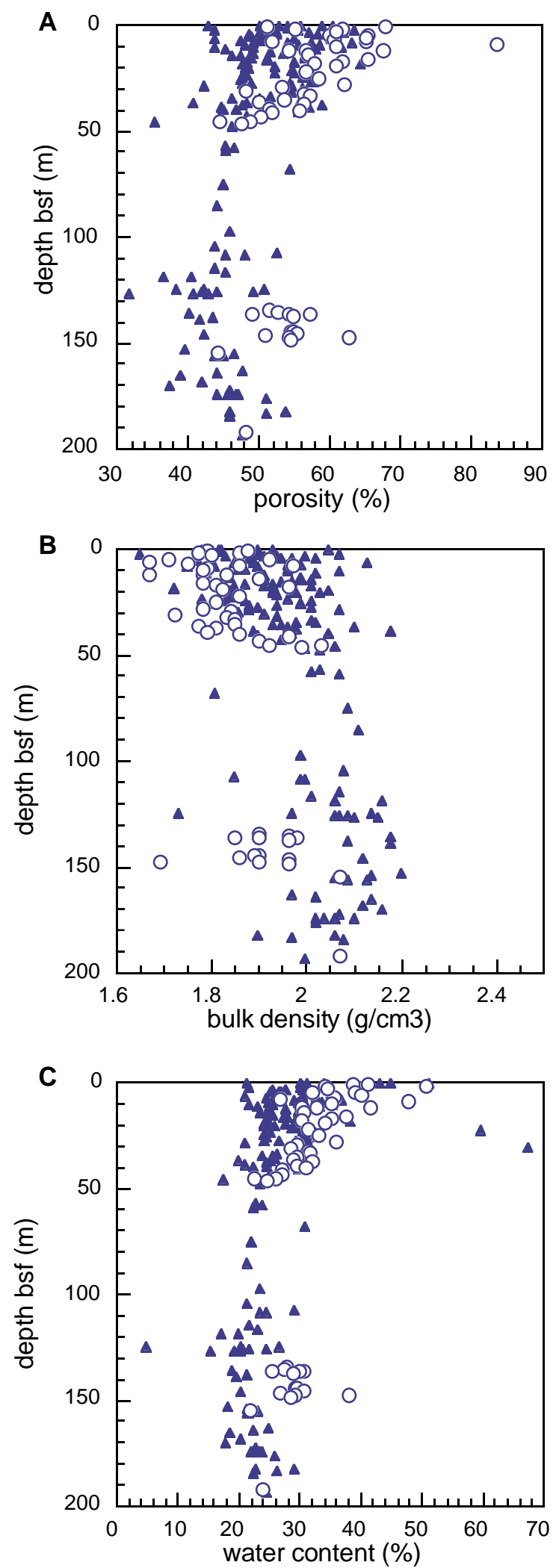

Figure 4. Shipboard physical properties data measured routinely during Leg 160 (Emeis, Robertson, Richter, et al., 1996). A. Porosity vs. depth. B. Wet bulk density vs. depth. C. Water content vs. depth. Mud-volcano deposits are represented by solid triangles; hemipelagic sediments are open circles. 
Table 3. Index properties and carbonate content of mud-volcano deposits.

\begin{tabular}{|c|c|c|c|c|c|c|c|c|c|}
\hline $\begin{array}{l}\text { Core, section, } \\
\text { interval }(\mathrm{cm})\end{array}$ & $\begin{array}{l}\text { Depth } \\
\text { (mbsf) }\end{array}$ & $\begin{array}{l}\text { Initial water } \\
\text { content (wt } \% \text { ) }\end{array}$ & $\begin{array}{l}\text { Porosity } \\
\text { (vol\%) }\end{array}$ & $\begin{array}{r}\text { Bulk d } \\
(\mathrm{g} / \mathrm{cl}\end{array}$ & $\begin{array}{l}\text { ensity } \\
\left.n^{3}\right)^{*}\end{array}$ & $\begin{array}{l}\text { Grain } \\
\quad(\mathrm{g} / \mathrm{cr}\end{array}$ & $\begin{array}{l}\text { ensity } \\
\left.3^{3}\right)^{*}\end{array}$ & $\begin{array}{r}\text { Dry d } \\
(\mathrm{g} / \mathrm{cl}\end{array}$ & $\begin{array}{l}\text { ensity } \\
\left.3^{3}\right)^{*}\end{array}$ \\
\hline Milano & & & & & & & & & \\
\hline $160-970 \mathrm{~A}-$ & & & & & & & & & \\
\hline $1 \mathrm{H}-1,71-73$ & 0.71 & 34.18 & 60.70 & 1.82 & 1.76 & 3.05 & 2.79 & 1.20 & 1.16 \\
\hline $1 \mathrm{H}-2,71-73$ & 2.21 & 30.50 & 54.17 & 1.82 & 1.81 & 2.76 & 2.72 & 1.26 & 1.26 \\
\hline $1 \mathrm{H}-3,70-72$ & 3.70 & 27.79 & 52.22 & 1.93 & 1.87 & 2.91 & 2.73 & 1.39 & 1.35 \\
\hline $1 \mathrm{H}-4,75-77$ & 5.25 & 27.10 & 51.46 & 1.95 & 1.88 & 2.92 & 2.72 & 1.42 & 1.37 \\
\hline $1 \mathrm{H}-5,61-63$ & 6.61 & 26.24 & 50.34 & 1.97 & 1.90 & 2.92 & 2.74 & 1.45 & 1.40 \\
\hline $2 \mathrm{H}-1,123-125$ & 8.83 & 37.08 & 63.24 & 1.75 & 1.68 & 2.99 & 2.71 & 1.10 & 1.06 \\
\hline $2 \mathrm{H}-2,90-92$ & 10.00 & 33.81 & 58.90 & 1.78 & 1.74 & 2.87 & 2.72 & 1.18 & 1.15 \\
\hline 3X-CC, $17-19$ & 10.37 & 25.13 & 48.05 & 1.96 & 1.91 & 2.82 & 2.70 & 1.47 & 1.43 \\
\hline $4 \mathrm{X}-1,90-92$ & 19.10 & 38.42 & 64.69 & 1.72 & 1.67 & 3.01 & 2.77 & 1.06 & 1.03 \\
\hline $6 \mathrm{X}-1,44-46$ & 37.84 & 19.97 & 41.02 & 2.10 & 2.05 & 2.86 & 2.74 & 1.68 & 1.64 \\
\hline $7 \mathrm{X}-1,10-12$ & 47.20 & 24.39 & 47.71 & 2.00 & 1.93 & 2.90 & 2.69 & 1.52 & 1.46 \\
\hline $10 \mathrm{X}-1,10-12$ & 76.10 & 22.20 & 45.18 & 2.09 & 2.00 & 2.96 & 2.75 & 1.62 & 1.56 \\
\hline $11 \mathrm{X}-\mathrm{CC}, 20-22$ & 85.90 & 21.44 & 44.13 & 2.11 & 2.03 & 2.97 & 2.76 & 1.66 & 1.59 \\
\hline $13 \mathrm{X}-1,40-42$ & 105.50 & 21.57 & 43.87 & 2.08 & 2.02 & 2.91 & 2.75 & 1.63 & 1.58 \\
\hline $14 \mathrm{X}-1,28-30$ & 115.08 & 21.76 & 43.89 & 2.07 & 1.99 & 2.88 & 2.69 & 1.62 & 1.55 \\
\hline $15 X-1,45-47$ & 124.85 & 26.58 & 51.04 & 1.97 & 1.91 & 2.95 & 2.77 & 1.44 & 1.40 \\
\hline $15 X-1,75-77$ & 125.15 & 20.26 & 42.39 & 2.14 & 2.04 & 2.97 & 2.72 & 1.71 & 1.62 \\
\hline $15 \mathrm{X}-1,126-128$ & 125.66 & 4.97 & 38.39 & 1.73 & 2.50 & 1.80 & 2.71 & 1.64 & 2.38 \\
\hline $15 \mathrm{X}-2,12-14$ & 125.91 & 21.91 & 44.16 & 2.06 & 2.00 & 2.89 & 2.72 & 1.61 & 1.56 \\
\hline $15 X-2,62-64$ & 126.41 & 20.68 & 42.17 & 2.09 & 2.03 & 2.87 & 2.73 & 1.66 & 1.61 \\
\hline $15 \mathrm{X}-2,102-104$ & 126.81 & 15.54 & 31.87 & 2.10 & 2.27 & 2.60 & 2.93 & 1.77 & 1.92 \\
\hline $15 \mathrm{X}-3,8-10$ & 127.37 & 20.46 & 42.99 & 2.15 & 2.05 & 3.00 & 2.76 & 1.71 & 1.63 \\
\hline $18 X-1,53-55$ & 153.73 & 18.40 & 39.59 & 2.20 & 2.10 & 2.98 & 2.75 & 1.80 & 1.71 \\
\hline $18 \mathrm{X}-1,100-102$ & 154.20 & 21.34 & 44.56 & 2.14 & 2.01 & 3.04 & 2.71 & 1.68 & 1.58 \\
\hline $18 \mathrm{X}-2,100-102$ & 155.50 & 23.22 & 46.61 & 2.06 & 1.97 & 2.96 & 2.74 & 1.58 & 1.52 \\
\hline $18 X-3,50-52$ & 156.50 & 21.48 & 43.87 & 2.09 & 2.02 & 2.93 & 2.75 & 1.64 & 1.59 \\
\hline $18 \mathrm{X}-3,100-102$ & 157.00 & 21.68 & 45.11 & 2.13 & 2.04 & 3.04 & 2.81 & 1.67 & 1.60 \\
\hline $19 X-1,66-68$ & 163.46 & 24.84 & 47.78 & 1.97 & 1.92 & 2.84 & 2.70 & 1.48 & 1.44 \\
\hline $19 \mathrm{X}-2,40-42$ & 164.70 & 22.44 & 44.32 & 2.02 & 2.00 & 2.82 & 2.75 & 1.57 & 1.55 \\
\hline $20 \mathrm{X}-1,70-72$ & 173.20 & 22.89 & 46.16 & 2.07 & 1.96 & 2.96 & 2.70 & 1.59 & 1.51 \\
\hline $20 X-2,49-51$ & 174.49 & 23.57 & 46.99 & 2.04 & 1.96 & 2.94 & 2.72 & 1.56 & 1.49 \\
\hline $20 \mathrm{X}-2,50-52$ & 174.50 & 24.01 & 47.26 & 2.02 & 1.94 & 2.91 & 2.70 & 1.53 & 1.47 \\
\hline $20 \mathrm{X}-2,100-102$ & 175.00 & 22.27 & 45.74 & 2.10 & 2.04 & 3.01 & 2.85 & 1.64 & 1.59 \\
\hline $21 X-1,50-52$ & 182.60 & 22.95 & 46.04 & 2.06 & 1.98 & 2.94 & 2.74 & 1.58 & 1.52 \\
\hline $21 \mathrm{X}-1,99-101$ & 183.09 & 29.06 & 54.03 & 1.90 & 1.85 & 2.94 & 2.75 & 1.35 & 1.31 \\
\hline $21 X-2,16-18$ & 183.76 & 26.51 & 51.08 & 1.97 & 1.89 & 2.97 & 2.71 & 1.45 & 1.39 \\
\hline $21 X-2,84-86$ & 184.44 & 22.68 & 46.09 & 2.08 & 2.02 & 2.99 & 2.82 & 1.61 & 1.56 \\
\hline 160-970C- & & & & & & & & & \\
\hline $1 \mathrm{H}-1,121-123$ & 1.21 & 30.18 & 52.89 & 1.80 & 1.81 & 2.66 & 2.69 & 1.25 & 1.26 \\
\hline $1 \mathrm{H}-2,124-126$ & 2.74 & 30.12 & 55.87 & 1.90 & 1.83 & 3.01 & 2.75 & 1.33 & 1.28 \\
\hline $1 \mathrm{H}-3,125-127$ & 4.25 & 27.86 & 52.76 & 1.94 & 1.88 & 2.96 & 2.79 & 1.40 & 1.36 \\
\hline $1 \mathrm{H}-4,10-12$ & 4.60 & 26.75 & 52.75 & 2.02 & 1.89 & 3.13 & 2.74 & 1.48 & 1.39 \\
\hline $2 \mathrm{H}-1,30-32$ & 5.50 & 25.34 & 48.72 & 1.97 & 1.90 & 2.87 & 2.68 & 1.47 & 1.42 \\
\hline $2 \mathrm{H}-2,36-38$ & 6.56 & 26.58 & 49.04 & 1.89 & 1.88 & 2.72 & 2.70 & 1.39 & 1.38 \\
\hline $2 \mathrm{H}-3,28-30$ & 7.90 & 26.45 & 50.51 & 1.96 & 1.92 & 2.91 & 2.80 & 1.44 & 1.41 \\
\hline $3 \mathrm{H}-1,132-134$ & 9.52 & 25.58 & 49.25 & 1.97 & 1.92 & 2.89 & 2.76 & 1.47 & 1.43 \\
\hline $3 \mathrm{H}-2,140-142$ & 11.10 & 25.72 & 49.30 & 1.96 & 1.90 & 2.88 & 2.69 & 1.46 & 1.41 \\
\hline $3 \mathrm{H}-3,90-92$ & 12.10 & 23.08 & 45.53 & 2.02 & 1.95 & 2.85 & 2.68 & 1.55 & 1.50 \\
\hline $3 \mathrm{H}-4,128-130$ & 13.98 & 25.44 & 49.41 & 1.99 & 1.94 & 2.93 & 2.79 & 1.48 & 1.45 \\
\hline $3 \mathrm{H}-5,91-93$ & 15.11 & 24.51 & 47.65 & 1.99 & 1.95 & 2.87 & 2.75 & 1.50 & 1.47 \\
\hline $3 \mathrm{H}-6,45-47$ & 15.72 & 25.35 & 48.68 & 1.97 & 1.91 & 2.86 & 2.69 & 1.47 & 1.42 \\
\hline 160-970D- & & & & & & & & & \\
\hline $1 \mathrm{H}-1,81-83$ & 0.81 & 21.53 & 43.13 & 2.05 & 2.03 & 2.83 & 2.77 & 1.61 & 1.59 \\
\hline $1 \mathrm{H}-2,129-131$ & 2.79 & 21.77 & 44.03 & 2.07 & 2.02 & 2.90 & 2.76 & 1.62 & 1.58 \\
\hline $1 \mathrm{H}-3,120-122$ & 4.20 & 25.76 & 50.23 & 2.00 & 1.92 & 2.98 & 2.77 & 1.48 & 1.43 \\
\hline $2 \mathrm{H}-1,50-52$ & 5.30 & 31.82 & 58.41 & 1.88 & 1.80 & 3.08 & 2.79 & 1.28 & 1.23 \\
\hline $2 \mathrm{H}-2,50-52$ & 6.80 & 30.61 & 55.16 & 1.85 & 1.82 & 2.86 & 2.78 & 1.28 & 1.27 \\
\hline $2 \mathrm{H}-4,46-48$ & 9.76 & 29.83 & 54.54 & 1.87 & 1.82 & 2.89 & 2.73 & 1.31 & 1.28 \\
\hline $2 \mathrm{H}-5,50-52$ & 11.30 & 21.66 & 43.79 & 2.07 & 2.00 & 2.89 & 2.71 & 1.62 & 1.57 \\
\hline $2 \mathrm{H}-6,50-52$ & 12.80 & 30.72 & 56.89 & 1.90 & 1.85 & 3.05 & 2.89 & 1.31 & 1.28 \\
\hline $3 \mathrm{H}-1,75-77$ & 15.05 & 30.47 & 56.79 & 1.91 & 1.81 & 3.07 & 2.73 & 1.33 & 1.26 \\
\hline $3 \mathrm{H}-2,75-77$ & 16.55 & 29.81 & 56.74 & 1.95 & 1.82 & 3.16 & 2.72 & 1.37 & 1.28 \\
\hline $3 \mathrm{H}-3,75-77$ & 18.05 & 30.58 & 55.27 & 1.85 & 1.82 & 2.87 & 2.78 & 1.29 & 1.27 \\
\hline $3 \mathrm{H}-5,75-77$ & 21.05 & 29.77 & 57.18 & 1.97 & 1.85 & 3.23 & 2.81 & 1.38 & 1.30 \\
\hline $3 \mathrm{H}-6,79-81$ & 22.59 & 30.04 & 56.92 & 1.94 & 1.81 & 3.15 & 2.70 & 1.36 & 1.27 \\
\hline $3 \mathrm{H}-7,43-45$ & 23.73 & 59.74 & 52.41 & 3.46 & 1.38 & -1.37 & 2.82 & 1.39 & 0.55 \\
\hline $4 \mathrm{H}-1,50-52$ & 24.30 & 32.80 & 57.10 & 1.78 & 1.77 & 2.79 & 2.74 & 1.20 & 1.19 \\
\hline $4 \mathrm{H}-2,50-52$ & 25.41 & 32.68 & 58.51 & 1.83 & 1.78 & 2.98 & 2.77 & 1.23 & 1.20 \\
\hline $4 \mathrm{H}-3,50-52$ & 26.91 & 30.54 & 57.73 & 1.94 & 1.83 & 3.18 & 2.80 & 1.35 & 1.27 \\
\hline $4 \mathrm{H}-4,50-52$ & 28.41 & 29.49 & 54.64 & 1.90 & 1.83 & 2.95 & 2.74 & 1.34 & 1.29 \\
\hline $4 \mathrm{H}-5,48-50$ & 29.89 & 31.12 & 56.41 & 1.86 & 1.80 & 2.93 & 2.73 & 1.28 & 1.24 \\
\hline $4 \mathrm{H}-6,50-52$ & 31.41 & 67.67 & 56.23 & 1.91 & 1.10 & -2.36 & 1.29 & 0.62 & 0.35 \\
\hline $5 \mathrm{H}-1,91-93$ & 34.21 & 26.45 & 51.91 & 2.01 & 1.92 & 3.08 & 2.79 & 1.48 & 1.41 \\
\hline $5 \mathrm{H}-2,79-81$ & 35.52 & 25.55 & 50.40 & 2.02 & 1.95 & 3.03 & 2.82 & 1.50 & 1.45 \\
\hline $5 \mathrm{H}-3,79-81$ & 36.79 & 29.73 & 56.07 & 1.93 & 1.83 & 3.09 & 2.74 & 1.36 & 1.29 \\
\hline $5 \mathrm{H}-4,78-80$ & 38.28 & 30.92 & 59.21 & 1.96 & 1.83 & 3.32 & 2.83 & 1.36 & 1.27 \\
\hline $5 \mathrm{H}-5,74-76$ & 39.74 & 30.97 & 57.27 & 1.89 & 1.79 & 3.06 & 2.71 & 1.31 & 1.24 \\
\hline $5 \mathrm{H}-6,82-84$ & 41.32 & 30.04 & 55.70 & $\begin{array}{l}1.09 \\
1.90\end{array}$ & 1.87 & 3.00 & 2.90 & 1.33 & $\begin{array}{l}1.24 \\
1.31\end{array}$ \\
\hline Napoli & & & & & & & & & \\
\hline $160-971 \mathrm{~A}-$ & & & & & & & & & \\
\hline $3 \mathrm{H}-1,68-70$ & 17.18 & 27.36 & 51.54 & 1.93 & 1.86 & 2.89 & 2.69 & 1.40 & 1.35 \\
\hline $3 \mathrm{H}-2,68-70$ & 18.68 & 24.74 & 48.55 & 2.01 & 1.93 & 2.94 & 2.71 & 1.51 & 1.45 \\
\hline $3 \mathrm{H}-3,68-70$ & 20.18 & 24.26 & 48.55 & 2.05 & 1.93 & 3.02 & 2.70 & 1.55 & 1.46 \\
\hline $3 \mathrm{H}-4,68-70$ & 21.68 & 24.93 & 48.14 & 1.98 & 1.94 & 2.86 & 2.76 & 1.49 & 1.46 \\
\hline $4 \mathrm{H}-1,128-130$ & 23.78 & 25.33 & 48.38 & 1.96 & 1.92 & 2.83 & 2.73 & 1.46 & 1.43 \\
\hline $4 \mathrm{H}-2,127-129$ & 25.27 & 24.44 & 47.97 & 2.01 & 1.95 & 2.92 & 2.75 & 1.52 & 1.47 \\
\hline
\end{tabular}


Table 3 (continued).

\begin{tabular}{|c|c|c|c|c|c|c|c|c|c|c|}
\hline \multirow{2}{*}{$\begin{array}{c}\begin{array}{c}\text { Core, section, } \\
\text { interval }(\mathrm{cm})\end{array} \\
4 \mathrm{H}-2,127-129\end{array}$} & \multirow{2}{*}{$\begin{array}{r}\begin{array}{r}\text { Depth } \\
(\mathrm{mbsf})\end{array} \\
25.27\end{array}$} & $\begin{array}{l}\text { Initial water } \\
\text { content (wt } \%)\end{array}$ & $\begin{array}{l}\text { Porosity } \\
\text { (vol\%) }\end{array}$ & $\begin{array}{r}\text { Bulk d } \\
(\mathrm{g} / \mathrm{cr}\end{array}$ & $\begin{array}{l}\text { ensity } \\
\left.n^{3}\right)^{*}\end{array}$ & $\begin{array}{r}\text { Grain } \mathrm{c} \\
\quad(\mathrm{g} / \mathrm{cn}\end{array}$ & $\begin{array}{l}\text { lensity } \\
\left.n^{3}\right)^{*}\end{array}$ & $\begin{array}{r}\text { Dry de } \\
(\mathrm{g} / \mathrm{cm}\end{array}$ & $\begin{array}{l}\text { ensity } \\
\left.n^{3}\right)^{*}\end{array}$ & $\begin{array}{r}\mathrm{CaCO}_{3} \\
(\mathrm{wt} \%)^{* *}\end{array}$ \\
\hline & & 24.44 & 47.97 & 2.01 & 1.95 & 2.92 & 2.75 & 1.52 & 1.47 & - \\
\hline $4 \mathrm{H}-3,119-121$ & 26.69 & 24.46 & 47.52 & 1.99 & 1.93 & 2.87 & 2.71 & 1.50 & 1.46 & 3.6 \\
\hline $4 \mathrm{H}-4,86-88$ & 27.86 & 24.43 & 47.90 & 2.01 & 1.95 & 2.91 & 2.75 & 1.52 & 1.47 & - \\
\hline 5X-CC, $35-37$ & 28.85 & 21.02 & 42.46 & 2.07 & 2.01 & 2.84 & 2.71 & 1.63 & 1.59 & - \\
\hline $6 X-1,19-21$ & 38.39 & 24.72 & 47.82 & 1.98 & 1.94 & 2.86 & 2.74 & 1.49 & 1.46 & - \\
\hline 6X-CC, $25-27$ & 39.16 & 21.02 & 44.74 & 2.18 & 2.00 & 3.12 & 2.69 & 1.72 & 1.58 & 38.7 \\
\hline $7 X-1,20-22$ & 48.10 & 23.41 & 46.33 & 2.03 & 1.99 & 2.89 & 2.80 & 1.55 & 1.52 & - \\
\hline $8 X-1,19-21$ & 57.79 & 22.86 & 45.40 & 2.03 & 2.01 & 2.87 & 2.81 & 1.57 & 1.55 & 15.2 \\
\hline $8 \mathrm{X}-\mathrm{CC}, 13-15$ & 58.15 & 23.81 & 46.78 & 2.01 & 1.93 & 2.88 & 2.68 & 1.53 & 1.47 & - \\
\hline 160-971B- & & & & & & & & & & \\
\hline $4 \mathrm{H}-1,50-52$ & 21.70 & 24.22 & 48.10 & 2.03 & 1.95 & 2.97 & 2.74 & 1.54 & 1.48 & 26.2 \\
\hline $6 X-1,25-27$ & 39.95 & 22.61 & 45.14 & 2.05 & 1.97 & 2.88 & 2.70 & 1.58 & 1.53 & 23.9 \\
\hline $7 X-1,50-52$ & 49.80 & 9.19 & 18.77 & 2.09 & 2.58 & 2.34 & 3.05 & 1.90 & 2.34 & - \\
\hline $8 X-1,53-55$ & 59.43 & 22.39 & 45.33 & 2.07 & 2.02 & 2.94 & 2.80 & 1.61 & 1.57 & 23.3 \\
\hline $9 X-1,27-29$ & 68.87 & 30.96 & 54.67 & 1.81 & 1.77 & 2.76 & 2.62 & 1.25 & 1.22 & 18 \\
\hline $12 \mathrm{X}-1,82-84$ & 98.32 & 23.71 & 45.94 & 1.99 & 1.92 & 2.80 & 2.65 & 1.51 & 1.47 & - \\
\hline $13 X-1,76-78$ & 107.86 & 29.11 & 52.6 & 1.85 & 1.80 & 2.77 & 2.62 & 1.31 & 1.28 & 21.7 \\
\hline $13 \mathrm{X}-2,38-40$ & 108.98 & 24.76 & 48.22 & 2.00 & 1.94 & 2.90 & 2.74 & 1.50 & 1.46 & - \\
\hline $13 \mathrm{X}-\mathrm{CC}, 6-8$ & 109.38 & 23.48 & 45.59 & 1.99 & 1.96 & 2.80 & 2.73 & 1.52 & 1.50 & - \\
\hline $14 X-1,92-94$ & 117.62 & 23.14 & 45.43 & 2.01 & 1.95 & 2.83 & 2.68 & 1.55 & 1.50 & - \\
\hline $14 \mathrm{X}-2,54-56$ & 118.74 & 20.21 & 40.69 & 2.06 & 2.03 & 2.78 & 2.69 & 1.65 & 1.62 & 23.2 \\
\hline $14 \mathrm{X}-\mathrm{CC}, 10-12$ & 119.38 & 17.36 & 36.61 & 2.16 & 2.11 & 2.82 & 2.72 & 1.79 & 1.74 & - \\
\hline $15 X-1,42-44$ & 126.72 & 24.50 & 49.38 & 2.07 & 1.95 & 3.08 & 2.75 & 1.56 & 1.47 & 21.1 \\
\hline $15 \mathrm{X}-\mathrm{CC}, 6-8$ & 127.52 & 19.52 & 40.92 & 2.15 & 2.08 & 2.93 & 2.77 & 1.73 & 1.67 & - \\
\hline $16 \mathrm{X}-1,50-52$ & 136.50 & 18.94 & 40.39 & 2.18 & 2.07 & 2.97 & 2.73 & 1.77 & 1.68 & 3.7 \\
\hline $16 \mathrm{X}-1,115-117$ & 137.15 & 12.92 & 28.75 & 2.28 & 2.23 & 2.78 & 2.70 & 1.98 & 1.94 & 3.7 \\
\hline $16 \mathrm{X}-2,58-60$ & 138.08 & 21.47 & 43.75 & 2.09 & 2.00 & 2.91 & 2.69 & 1.64 & 1.57 & 12.7 \\
\hline $16 X-3,50-52$ & 139.44 & 19.60 & 41.69 & 2.18 & 2.08 & 3.01 & 2.78 & 1.75 & 1.67 & 11.2 \\
\hline $17 \mathrm{X}-1,60-62$ & 146.20 & 20.54 & 42.56 & 2.12 & 2.06 & 2.94 & 2.78 & 1.69 & 1.63 & 10 \\
\hline $19 X-1,50-52$ & 165.40 & 18.66 & 39.01 & 2.14 & 2.07 & 2.86 & 2.70 & 1.74 & 1.68 & 19.5 \\
\hline $19 X-3,50-52$ & 168.40 & 20.41 & 42.14 & 2.12 & 2.04 & 2.91 & 2.74 & 1.68 & 1.63 & 15.5 \\
\hline $19 X-5,50-52$ & 171.09 & 17.86 & 37.70 & 2.16 & 2.10 & 2.85 & 2.72 & 1.78 & 1.72 & 17.6 \\
\hline $20 X-1,50-52$ & 175.00 & 22.02 & 44.24 & 2.06 & 1.99 & 2.88 & 2.71 & 1.60 & 1.55 & 19.3 \\
\hline $20 X-2,50-52$ & 176.40 & 25.98 & 51.31 & 2.02 & 1.91 & 3.08 & 2.75 & 1.50 & 1.42 & 20.6 \\
\hline $22 \mathrm{X}-1,15-17$ & 193.95 & 24.58 & 47.92 & 2.00 & 1.93 & 2.89 & 2.70 & 1.51 & 1.45 & - \\
\hline $22 \mathrm{X}-\mathrm{C}, 24-26$ & 194.30 & 6.87 & 16.88 & 2.52 & 2.42 & 2.82 & 2.70 & 2.34 & 2.26 & - \\
\hline 160-971D- & & & & & & & & & & \\
\hline $1 \mathrm{H}-2,116-118$ & 2.66 & 39.35 & 63.53 & 1.65 & 1.61 & 2.75 & 2.57 & 1.00 & 0.98 & 28.2 \\
\hline $1 \mathrm{H}-3,116-118$ & 4.16 & 34.69 & 62.01 & 1.83 & 1.71 & 3.15 & 2.65 & 1.20 & 1.12 & - \\
\hline $1 \mathrm{H}-4,116-118$ & 5.66 & 35.23 & 61.03 & 1.77 & 1.69 & 2.95 & 2.60 & 1.15 & 1.09 & - \\
\hline $1 \mathrm{H}-5,124-126$ & 7.24 & 21.13 & 43.93 & 2.13 & 2.00 & 3.00 & 2.69 & 1.68 & 1.58 & 25.5 \\
\hline $1 \mathrm{H}-6,21-23$ & 7.71 & 24.59 & 47.70 & 1.99 & 1.90 & 2.87 & 2.63 & 1.50 & 1.43 & - \\
\hline $2 \mathrm{H}-1,126-128$ & 9.26 & 29.25 & 54.35 & 1.90 & 1.84 & 2.95 & 2.74 & 1.35 & 1.30 & - \\
\hline $2 \mathrm{H}-2,128-130$ & 10.78 & 35.45 & 61.79 & 1.79 & 1.70 & 3.02 & 2.66 & 1.15 & 1.10 & 24.8 \\
\hline $2 \mathrm{H}-3,131-133$ & 12.31 & 33.40 & 58.23 & 1.79 & 1.72 & 2.85 & 2.61 & 1.19 & 1.15 & - \\
\hline $2 \mathrm{H}-4,133-135$ & 13.83 & 30.01 & 53.78 & 1.84 & 1.78 & 2.78 & 2.60 & 1.29 & 1.25 & - \\
\hline $2 \mathrm{H}-5,128-130$ & 15.28 & 23.72 & 46.44 & 2.01 & 1.93 & 2.86 & 2.67 & 1.53 & 1.48 & 23.8 \\
\hline $2 \mathrm{H}-6,134-136$ & 16.84 & 27.99 & 51.20 & 1.87 & 1.83 & 2.77 & 2.63 & 1.35 & 1.32 & - \\
\hline $3 \mathrm{H}-1,117-119$ & 18.67 & 29.61 & 55.12 & 1.91 & 1.82 & 2.99 & 2.71 & 1.34 & 1.28 & - \\
\hline $3 \mathrm{H}-2,128-130$ & 20.28 & 29.28 & 52.54 & 1.84 & 1.81 & 2.74 & 2.64 & 1.30 & 1.28 & 24.4 \\
\hline $3 \mathrm{H}-3,128-130$ & 21.78 & 28.98 & 54.86 & 1.94 & 1.83 & 3.05 & 2.69 & 1.38 & 1.30 & - \\
\hline $3 \mathrm{H}-4,122-124$ & 23.22 & 30.70 & 55.09 & 1.84 & 1.79 & 2.84 & 2.67 & 1.27 & 1.24 & - \\
\hline $3 \mathrm{H}-5,129-131$ & 24.79 & 30.82 & 56.40 & 1.87 & 1.80 & 2.97 & 2.70 & 1.30 & 1.24 & 22.7 \\
\hline $3 \mathrm{H}-6,120-122$ & 26.20 & 32.97 & 58.11 & 1.81 & 1.74 & 2.89 & 2.67 & 1.21 & 1.17 & - \\
\hline $3 \mathrm{H}-7,63-65$ & 27.13 & 31.30 & 57.58 & 1.88 & 1.78 & 3.05 & 2.68 & 1.29 & 1.22 & - \\
\hline $4 \mathrm{H}-1,130-132$ & 28.30 & 26.69 & 48.98 & 1.88 & 1.83 & 2.70 & 2.57 & 1.38 & 1.34 & - \\
\hline $4 \mathrm{H}-2,129-131$ & 29.79 & 29.81 & 54.70 & 1.88 & 1.80 & 2.91 & 2.65 & 1.32 & 1.26 & 23.7 \\
\hline $4 \mathrm{H}-3,135-137$ & 31.35 & 29.96 & 54.19 & 1.85 & 1.78 & 2.83 & 2.61 & 1.30 & 1.25 & - \\
\hline $4 \mathrm{H}-4,119-121$ & 32.69 & 29.02 & 54.87 & 1.94 & 1.83 & 3.05 & 2.70 & 1.37 & 1.30 & 24.2 \\
\hline $4 \mathrm{H}-6,132-134$ & 35.82 & 23.94 & 46.28 & 1.98 & 1.91 & 2.80 & 2.61 & 1.51 & 1.45 & - \\
\hline $4 \mathrm{H}-7,71-73$ & 36.71 & 26.04 & 49.61 & 1.95 & 1.88 & 2.87 & 2.67 & 1.44 & 1.39 & - \\
\hline $5 \mathrm{H}-1,50-52$ & 37.00 & 25.27 & 48.42 & 1.96 & 1.88 & 2.84 & 2.62 & 1.47 & 1.40 & - \\
\hline $5 \mathrm{H}-3,70-72$ & 40.20 & 24.49 & 46.99 & 1.97 & 1.90 & 2.80 & 2.63 & 1.48 & 1.43 & 24.2 \\
\hline $5 \mathrm{H}-5,50-52$ & 43.00 & 25.50 & 48.60 & 1.95 & 1.88 & 2.83 & 2.63 & 1.45 & 1.40 & 23.5 \\
\hline $5 \mathrm{H}-7,48-50$ & 45.98 & 17.73 & 35.58 & 2.06 & 1.98 & 2.63 & 2.48 & 1.69 & 1.63 & - \\
\hline $160-971 \mathrm{E}-$ & & & & & & & & & & \\
\hline $1 \mathrm{H}-1,75-77$ & 0.75 & 31.46 & 59.14 & 1.93 & 1.80 & 3.23 & 2.76 & 1.32 & 1.23 & 18.5 \\
\hline $1 \mathrm{H}-3,75-77$ & 3.75 & 30.67 & 57.31 & 1.91 & 1.80 & 3.11 & 2.70 & 1.33 & 1.25 & 29.1 \\
\hline $1 \mathrm{H}-5,72-74$ & 6.72 & 32.61 & 60.19 & 1.89 & 1.76 & 3.20 & 2.70 & 1.27 & 1.19 & 20.5 \\
\hline $1 \mathrm{H}-7,49-51$ & 9.49 & 32.56 & 57.58 & 1.81 & 1.75 & 2.88 & 2.66 & 1.22 & 1.18 & - \\
\hline $2 \mathrm{H}-1,65-67$ & 10.15 & 30.82 & 55.84 & 1.86 & 1.77 & 2.91 & 2.63 & 1.28 & 1.23 & - \\
\hline $2 \mathrm{H}-3,65-67$ & 13.15 & 27.82 & 51.79 & 1.91 & 1.83 & 2.86 & 2.64 & 1.38 & 1.32 & 23.9 \\
\hline $2 \mathrm{H}-5,65-67$ & 16.15 & 25.62 & 49.16 & 1.97 & 1.90 & 2.88 & 2.70 & 1.46 & 1.41 & 24.5 \\
\hline $2 \mathrm{H}-7,57-59$ & 18.97 & 25.01 & 48.00 & 1.97 & 1.90 & 2.83 & 2.65 & 1.47 & 1.42 & - \\
\hline $3 \mathrm{H}-1,73-75$ & 19.73 & 27.90 & 52.55 & 1.93 & 1.84 & 2.93 & 2.65 & 1.39 & 1.32 & 26.6 \\
\hline $3 \mathrm{H}-7,30-32$ & 21.49 & 25.93 & 48.83 & 1.93 & 1.89 & 2.79 & 2.69 & 1.43 & 1.40 & 25.1 \\
\hline Bannock & & & & & & & & & & \\
\hline BAN 89-02 & 0.8 & 45 & 54.3 & 1.818 & 1.793 & 2.743 & 2.743 & 1.254 & 1.258 & 34.3 \\
\hline BAN 89-04 & 1.2 & 43.2 & 50.4 & 1.9 & 1.916 & 2.675 & 2.675 & 1.327 & 1.417 & 24.8 \\
\hline MONZA & & & & & & & & & & \\
\hline Ban & & & & & & & & & & \\
\hline BAN 88-07 & 1.5 & 51.2 & 56.1 & 1.779 & 1.813 & 2.648 & 2.648 & 1.177 & 1.248 & 22.4 \\
\hline
\end{tabular}

Notes: * = two different methods used for all density measurements during Leg 160 (see Emeis, Robertson, Richter, et al., 1996); ** = sample taken from the same section. Bannock samples are axial and transversal trims. ${ }^{* *}=$ Sample taken from the same section. 
Table 4. Index properties and carbonate content of mixed sediment and nannofossil ooze.

\begin{tabular}{|c|c|c|c|c|c|c|c|c|c|c|}
\hline \multirow{2}{*}{$\begin{array}{c}\text { Core, section, } \\
\text { interval }(\mathrm{cm})\end{array}$} & \multirow{2}{*}{$\begin{array}{l}\text { Depth } \\
\text { (mbsf) }\end{array}$} & \multirow[t]{2}{*}{$\begin{array}{c}\text { Initial water } \\
\text { content (wt } \%)\end{array}$} & \multirow[t]{2}{*}{$\begin{array}{c}\text { Porosity } \\
(\operatorname{vol} \%)\end{array}$} & \multicolumn{2}{|c|}{$\begin{array}{l}\text { Bulk density } \\
\left(\mathrm{g} / \mathrm{cm}^{3}\right)^{*}\end{array}$} & \multicolumn{2}{|c|}{$\begin{array}{l}\text { Grain density } \\
\left(\mathrm{g} / \mathrm{cm}^{3}\right)^{*}\end{array}$} & \multicolumn{2}{|c|}{$\begin{array}{l}\text { Dry density } \\
\left(\mathrm{g} / \mathrm{cm}^{3}\right)^{*}\end{array}$} & \multirow[t]{2}{*}{$\begin{array}{c}\mathrm{CaCO}^{3} \\
(\mathrm{wt} \%)^{* *}\end{array}$} \\
\hline & & & & & & & & & & \\
\hline Milano & & & & & & & & & & \\
\hline $\begin{array}{l}\text { 160-970A- } \\
16 X-1,6163 \\
16 X-1,110112 \\
16 X-2,4042 \\
16 X-2,8082 \\
16 X-2,110112 \\
16 X-3,4547 \\
17 X-1,5052 \\
17 X-1,100102 \\
17 X-2,5052 \\
17 X-2,100102 \\
17 X-3,5052 \\
17 X-3,110112 \\
17 X-4,5052\end{array}$ & $\begin{array}{l}134.61 \\
135.10 \\
135.90 \\
136.30 \\
136.60 \\
137.45 \\
144.10 \\
144.60 \\
145.60 \\
146.10 \\
147.10 \\
147.70 \\
148.60\end{array}$ & $\begin{array}{l}27.68 \\
27.57 \\
25.34 \\
30.74 \\
30.06 \\
28.79 \\
29.37 \\
29.65 \\
30.51 \\
26.63 \\
38.09 \\
29.28 \\
28.55\end{array}$ & $\begin{array}{l}51.41 \\
52.81 \\
49.01 \\
57.14 \\
54.21 \\
54.96 \\
54.55 \\
54.74 \\
55.51 \\
50.88 \\
62.79 \\
54.32 \\
54.66\end{array}$ & $\begin{array}{l}1.90 \\
1.96 \\
1.98 \\
1.90 \\
1.85 \\
1.96 \\
1.90 \\
1.89 \\
1.86 \\
1.96 \\
1.69 \\
1.90 \\
1.96\end{array}$ & $\begin{array}{l}1.86 \\
1.86 \\
1.93 \\
1.81 \\
1.81 \\
1.83 \\
1.84 \\
1.82 \\
1.81 \\
1.88 \\
1.64 \\
1.83 \\
1.84\end{array}$ & $\begin{array}{l}2.83 \\
3.01 \\
2.90 \\
3.08 \\
2.82 \\
3.09 \\
2.96 \\
2.94 \\
2.91 \\
2.92 \\
2.81 \\
2.94 \\
3.09\end{array}$ & $\begin{array}{l}2.70 \\
2.71 \\
2.76 \\
2.74 \\
2.70 \\
2.69 \\
2.74 \\
2.71 \\
2.72 \\
2.71 \\
2.59 \\
2.71 \\
2.71\end{array}$ & $\begin{array}{l}1.38 \\
1.42 \\
1.48 \\
1.32 \\
1.29 \\
1.39 \\
1.34 \\
1.33 \\
1.30 \\
1.44 \\
1.05 \\
1.34 \\
1.40\end{array}$ & $\begin{array}{l}1.34 \\
1.35 \\
1.44 \\
1.25 \\
1.27 \\
1.31 \\
1.30 \\
1.28 \\
1.26 \\
1.38 \\
1.01 \\
1.29 \\
1.32\end{array}$ & $\begin{array}{l}\overline{-} \\
49.7 \\
49.7 \\
49.7 \\
47.1 \\
52.6 \\
52.6 \\
45.6 \\
45.6 \\
49.1 \\
49.1 \\
-\end{array}$ \\
\hline $\begin{array}{l}\text { 160-970B- } \\
1 \mathrm{H}-1,5355 \\
1 \mathrm{H}-2,5254 \\
1 \mathrm{H}-4,5557 \\
1 \mathrm{H}-6,5557 \\
2 \mathrm{H}-3,123125 \\
2 \mathrm{H}-4,4648 \\
2 \mathrm{H}-5,5860 \\
2 \mathrm{H}-6,8890 \\
2 \mathrm{H}-7,103105 \\
2 \mathrm{H}-8,7880 \\
3 \mathrm{H}-1,5254 \\
3 \mathrm{H}-3,5052 \\
3 \mathrm{H}-5,4951 \\
3 \mathrm{H}-7,5052 \\
4 \mathrm{H}-1,125127 \\
4 \mathrm{H}-2,126128 \\
4 \mathrm{H}-3,104106 \\
4 \mathrm{H}-4,3436 \\
4 \mathrm{H}-5,124126 \\
4 \mathrm{H}-6,1517 \\
4 \mathrm{H}-7,2224 \\
5 \mathrm{H}-1,108110 \\
5 \mathrm{H}-2,141143 \\
5 \mathrm{H}-3,100102 \\
5 \mathrm{H}-4,128130 \\
5 \mathrm{H}-5,124126 \\
5 \mathrm{H}-6,6668 \\
5 \mathrm{H}-7,1012\end{array}$ & $\begin{array}{r}0.53 \\
2.02 \\
5.05 \\
8.05 \\
11.86 \\
12.55 \\
14.05 \\
15.80 \\
17.35 \\
18.53 \\
19.52 \\
22.50 \\
25.49 \\
28.50 \\
29.75 \\
31.26 \\
32.54 \\
33.34 \\
35.74 \\
36.15 \\
37.72 \\
39.08 \\
40.64 \\
41.73 \\
43.51 \\
44.97 \\
45.89 \\
46.33\end{array}$ & $\begin{array}{l}38.76 \\
34.02 \\
32.01 \\
35.87 \\
30.39 \\
32.69 \\
30.71 \\
37.75 \\
35.03 \\
30.30 \\
34.24 \\
31.28 \\
33.24 \\
35.79 \\
29.59 \\
28.59 \\
31.45 \\
31.68 \\
29.61 \\
28.98 \\
31.92 \\
29.42 \\
30.83 \\
27.05 \\
27.10 \\
22.53 \\
26.01 \\
24.60\end{array}$ & $\begin{array}{l}67.84 \\
61.87 \\
59.98 \\
65.01 \\
54.28 \\
56.76 \\
56.96 \\
65.53 \\
61.95 \\
57.82 \\
60.86 \\
56.80 \\
58.61 \\
62.22 \\
53.19 \\
48.05 \\
56.29 \\
57.20 \\
53.50 \\
50.03 \\
56.39 \\
51.46 \\
55.88 \\
51.75 \\
50.37 \\
44.65 \\
48.75 \\
47.68\end{array}$ & $\begin{array}{l}1.79 \\
1.86 \\
1.92 \\
1.86 \\
1.83 \\
1.78 \\
1.90 \\
1.78 \\
1.81 \\
1.96 \\
1.82 \\
1.86 \\
1.81 \\
1.78 \\
1.84 \\
1.72 \\
1.83 \\
1.85 \\
1.85 \\
1.77 \\
1.81 \\
1.79 \\
1.86 \\
1.96 \\
1.90 \\
2.03 \\
1.92 \\
1.99\end{array}$ & $\begin{array}{l}1.65 \\
1.74 \\
1.79 \\
1.73 \\
1.81 \\
1.76 \\
1.80 \\
1.67 \\
1.72 \\
1.82 \\
1.72 \\
1.78 \\
1.75 \\
1.72 \\
1.82 \\
1.83 \\
1.78 \\
1.78 \\
1.86 \\
1.79 \\
1.77 \\
1.79 \\
1.79 \\
1.91 \\
1.85 \\
1.98 \\
1.87 \\
1.90\end{array}$ & $\begin{array}{l}3.42 \\
3.22 \\
3.26 \\
3.40 \\
2.79 \\
2.77 \\
3.06 \\
3.21 \\
3.09 \\
3.23 \\
3.06 \\
2.96 \\
2.91 \\
3.03 \\
2.77 \\
2.37 \\
2.88 \\
2.95 \\
2.80 \\
2.51 \\
2.82 \\
2.61 \\
2.91 \\
2.96 \\
2.80 \\
2.84 \\
2.77 \\
2.86\end{array}$ & $\begin{array}{l}2.68 \\
2.72 \\
2.77 \\
2.80 \\
2.71 \\
2.69 \\
2.72 \\
2.68 \\
2.71 \\
2.74 \\
2.64 \\
2.69 \\
2.70 \\
2.75 \\
2.70 \\
2.67 \\
2.70 \\
2.72 \\
2.84 \\
2.57 \\
2.67 \\
2.59 \\
2.68 \\
2.81 \\
2.64 \\
2.73 \\
2.64 \\
2.63\end{array}$ & $\begin{array}{l}1.10 \\
1.23 \\
1.31 \\
1.19 \\
1.27 \\
1.20 \\
1.32 \\
1.11 \\
1.18 \\
1.36 \\
1.20 \\
1.28 \\
1.21 \\
1.14 \\
1.30 \\
1.23 \\
1.26 \\
1.26 \\
1.30 \\
1.26 \\
1.23 \\
1.26 \\
1.28 \\
1.43 \\
1.39 \\
1.57 \\
1.42 \\
1.50\end{array}$ & $\begin{array}{l}1.01 \\
1.15 \\
1.22 \\
1.11 \\
1.26 \\
1.18 \\
1.25 \\
1.04 \\
1.12 \\
1.27 \\
1.13 \\
1.22 \\
1.17 \\
1.10 \\
1.28 \\
1.31 \\
1.22 \\
1.22 \\
1.31 \\
1.27 \\
1.20 \\
1.26 \\
1.24 \\
1.39 \\
1.35 \\
1.54 \\
1.39 \\
1.43\end{array}$ & $\begin{array}{l}52.1 \\
45.1 \\
55.7 \\
- \\
68.7 \\
56 \\
46.6 \\
46.7 \\
- \\
- \\
- \\
40.7 \\
56.6 \\
41.2 \\
36.4 \\
56 \\
54.6 \\
14.1 \\
53.8 \\
45.1 \\
14.5 \\
49.2 \\
49.1 \\
30.1 \\
43.8 \\
-\end{array}$ \\
\hline $\begin{array}{l}\text { Napoli } \\
160-971 \mathrm{~A}- \\
2 \mathrm{H}-1,5052 \\
2 \mathrm{H}-2,5052 \\
2 \mathrm{H}-3,5052 \\
2 \mathrm{H}-4,5052\end{array}$ & $\begin{array}{r}7.50 \\
9.00 \\
10.50 \\
12.00\end{array}$ & $\begin{array}{l}35.49 \\
47.77 \\
35.09 \\
41.38\end{array}$ & $\begin{array}{l}60.64 \\
83.69 \\
60.83 \\
67.58\end{array}$ & $\begin{array}{l}1.75 \\
1.79 \\
1.78 \\
1.67\end{array}$ & $\begin{array}{l}1.71 \\
1.45 \\
1.74 \\
1.61\end{array}$ & $\begin{array}{l}2.87 \\
5.74 \\
2.94 \\
3.03\end{array}$ & $\begin{array}{l}2.71 \\
2.32 \\
2.78 \\
2.71\end{array}$ & $\begin{array}{l}1.13 \\
0.94 \\
1.15 \\
0.98\end{array}$ & $\begin{array}{l}1.10 \\
0.75 \\
1.13 \\
0.94\end{array}$ & $\begin{array}{l}\overline{3} .9 \\
- \\
-\end{array}$ \\
\hline $\begin{array}{l}\text { Hilo } \\
\text { Bannock } \\
\text { BAN 89-08 }\end{array}$ & 2.1 & 50.7 & 55.2 & 1.772 & 1.752 & 2.628 & 2.628 & 1.176 & 1.172 & 65 \\
\hline Transition zone; $\mathrm{n}$ & ed sedi & & & & & & & & & \\
\hline $\begin{array}{l}\text { Milano } \\
\text { 160-970A- } \\
\text { 18X-2, 50 } 52 \\
\text { 22X-1, 50 52 }\end{array}$ & $\begin{array}{l}155.00 \\
192.20\end{array}$ & $\begin{array}{l}21.84 \\
23.84\end{array}$ & $\begin{array}{l}44.16 \\
48.06\end{array}$ & $\begin{array}{l}2.07 \\
2.07\end{array}$ & $\begin{array}{l}2.01 \\
1.96\end{array}$ & $\begin{array}{l}2.90 \\
3.03\end{array}$ & $\begin{array}{l}2.74 \\
2.75\end{array}$ & $\begin{array}{l}1.62 \\
1.57\end{array}$ & $\begin{array}{l}1.57 \\
1.49\end{array}$ & $\begin{array}{l}23.1 \\
51.1\end{array}$ \\
\hline $\begin{array}{l}\text { Napoli } \\
\text { 160-971A- } \\
\text { 2H-5, } 5052 \\
\text { 2H-6, } 5052 \\
\text { 2H-7, } 5052\end{array}$ & $\begin{array}{l}13.50 \\
15.00 \\
16.50\end{array}$ & $\begin{array}{l}34.63 \\
39.10 \\
40.08\end{array}$ & $\begin{array}{l}60.85 \\
65.44 \\
65.17\end{array}$ & $\begin{array}{l}1.80 \\
1.71 \\
1.67\end{array}$ & $\begin{array}{l}1.74 \\
1.65 \\
1.63\end{array}$ & $\begin{array}{l}3.01 \\
3.02 \\
2.87\end{array}$ & $\begin{array}{l}2.76 \\
2.70 \\
2.69\end{array}$ & $\begin{array}{l}1.18 \\
1.04 \\
1.00\end{array}$ & $\begin{array}{l}1.14 \\
1.00 \\
0.98\end{array}$ & $\overline{37.1}$ \\
\hline $\begin{array}{l}\text { 160-971B- } \\
3 \mathrm{H}-5,5052\end{array}$ & 18.20 & 26.88 & 51.80 & 1.97 & 1.92 & 2.99 & 2.82 & 1.44 & 1.40 & 23.3 \\
\hline $\begin{array}{l}\text { Bannock } \\
\text { BAN 89-03 }\end{array}$ & 1.1 & 41.2 & 51.3 & 1.877 & 1.882 & 2.727 & 2.727 & 1.329 & 1.35 & 37.8 \\
\hline
\end{tabular}

Notes: $*$ = two different methods used for all density measurements during Leg 160 (Emeis, Robertson, Richter, et al., 1996); ** = sample taken from the same section. Bannock samples are axial and transversal trims.

\section{Shear Strength of Sediments}

\section{Vane Shear Strength}

Routine measurements during Leg 160 provided only limited data from Sites 970 and 971 (see Emeis, Robertson, Richter, et al., 1996).
For the uppermost part of the sedimentary succession (0-20 mbsf), undrained (vane) shear strength is $5-45 \mathrm{kPa}$ and increases to $65-75$ $\mathrm{kPa}$ between 30 and 80 mbsf (Fig. 6). Mud breccias below $100 \mathrm{mbsf}$ (found at Holes 970A and 971B) were too firm for use of the vane apparatus. Shear strength measurements on non-mud volcanic deposits 
range from 13 to $50 \mathrm{KPa}$ at the very top to up to $120 \mathrm{KPa}$ at depths around $120 \mathrm{mbsf}$ and deeper (see Kopf et al., Chap. 49, this volume). The maximum values were dominantly obtained from sapropel layers, ranging between 120 and $150 \mathrm{kPa}$, while nannofossil ooze and clay generally range from 70 to $100 \mathrm{kPa}$. In general, a trend of increasing strength with depth can be seen.

\section{Results from Shear Box Tests of Leg 160 and Bannock Samples}

Peak and residual shear strengths were determined from mud-volcano deposits, transitional sediment, and hemipelagic sediments of the region (see also Kopf et al., Chap. 49, this volume), and are listed in Tables 1 and 6. Drained peak strengths from Leg 160 whole-round samples under 1 MPa vertical load varied from 199 to $399 \mathrm{kPa}$, giving friction angles of $18^{\circ}-30^{\circ} \pm 1^{\circ}$ (Table 1). Drained residual strengths of $155-338 \mathrm{kPa}$ were obtained, significantly higher than those using the vane apparatus (probably because the vane device tends to crack the sediment even when not indurated; see above). Shear-induced consolidation was considerable. Initial water contents of the mud-volcano deposits $(32.1 \%-42.4 \%)$ were reduced to $21.4 \%-30.6 \%$ after shearing (see Tables 3, 4). For the nannofossil ooze, a peak strength of $621 \mathrm{kPa}$ and a residual strength of $<580 \mathrm{kPa}$ were obtained under the same test conditions, giving angles of internal friction $30^{\circ} \pm 1^{\circ}$ and $<28^{\circ} \pm 1^{\circ}$.

The undisturbed near-surface mud-volcano sample sheared at lower effective stress (Table 6) shows a friction angle very similar to the shallowest remolded Leg 160 samples sheared at higher effective stress, with very small difference between peak and residual strength, thus suggesting that undisturbed samples of the mud-volcano sediments behave mechanically as remolded samples. The undisturbed transitional sample provided a higher friction angle than the remolded Leg 160 sample even with at lower effective stress.

Microfabrics observed after Leg 160 WR sample shear tests showed frequent sets of Riedel shear bands along the main shear zone. In addition, an enrichment of clay-grade nannofossil ooze was observed in the pressure shadows of well-indurated mudstone clasts (0.5-3 $\mathrm{mm}$ across), along the main shear surface between the two halves of the shear box, or along shear bands within the sample (Fig. 7). Scanning electron microscope study of the shear zone and fabrics revealed strong striations along the plane of failure. Both clasts and clay minerals (chlorite and presumably smectite; see paragraph on XRD results of fine-grained components above) have a preferred orientation with long axes parallel to the direction of movement. This texture is penetrative in interval 160-971B-16X-03, 99-119 cm, but is less obvious in the transitional sediment (interval 160-971B-4H$02,132-150 \mathrm{~cm}$ ). This observation, as well as the enrichment of fine carbonate mud (predominantly nannofossils) along shear bands and around clasts might be partly explained by differences in mineralogy and grain size. From Tables 3 and 4 it can be seen that initial (in situ) porosities for hemipelagic ooze and mud-volcano deposits are very similar at shallow depths. Grain-size analysis of the nannofossil ooze recorded only the clay and silt fractions (Tables 1,6). Thus, after compaction, particle bonding in the oozes is assumed to be less strong than in clay-rich mud-debris flow deposits. As a result, calcareous mud is mobile during shearing and particulate flow occurs to regions of diminished stress (see above and Fig. 7; e.g., Borradaile, 1981; Knipe, 1986; Agar, 1990).

\section{Consolidation and Permeability}

Consolidation characteristics of the Bannock gravity core samples of mud-volcano sediments are of naturally remolded soils. The consolidation curves of vertical and horizontal trims are in fact very similar to the laboratory remolded specimen, with a transition between the pre-compression and linear compression (on semi-log scale) that
Natural water contents and Atterberg limits (\%)

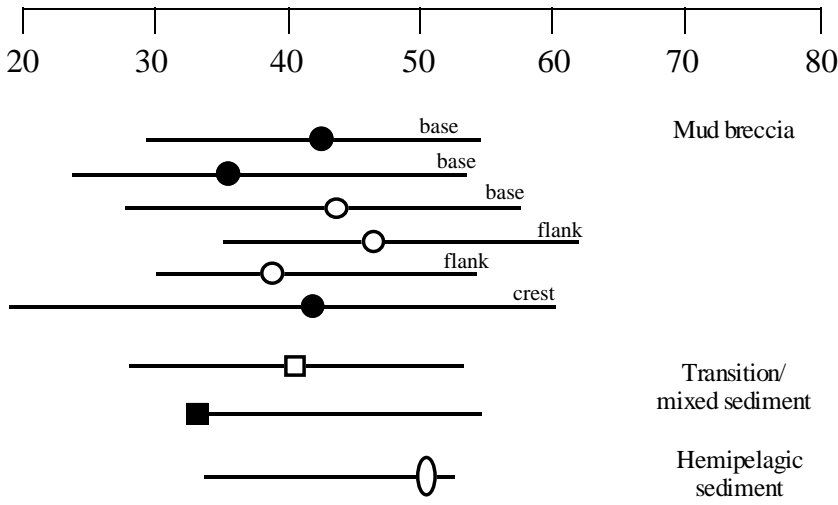

Figure 5. Natural water contents and Atterberg limits of samples from Leg 160 and Bannock cruises (see Table 5). Closed symbols indicate Leg 160 whole-round samples, open symbols are BAN specimens. Circles are mudvolcano deposits, squares are mixed sediment, and ellipses are hemipelagic ooze.
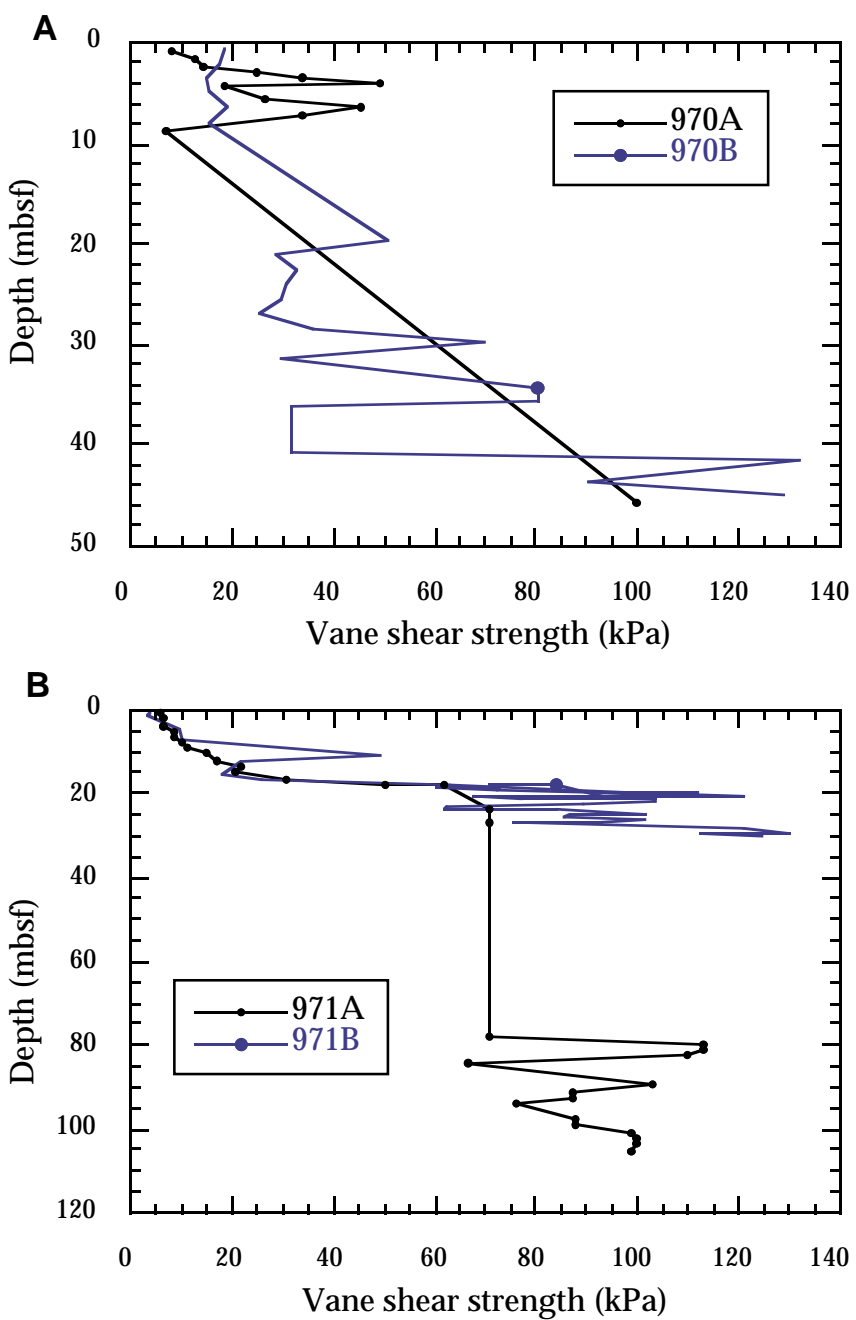

Figure 6. Summary of results from vane shear strength obtained routinely during Leg 160 from (A) Site 970 (Milano) and (B) Site 971 (Napoli). 
Table 5. Water contents and Atterberg limits.

\begin{tabular}{|c|c|c|c|c|c|c|}
\hline Core, section, interval (cm) & $\begin{array}{l}\text { Initial water } \\
\text { content }(\%)\end{array}$ & $\begin{array}{c}\text { Post-shear water } \\
\text { content }(\%)\end{array}$ & $\begin{array}{l}\text { Post-permeability } \\
\text { water content }(\%)\end{array}$ & $\begin{array}{l}\text { Liquid limit } \\
\text { (\%) }\end{array}$ & $\begin{array}{c}\text { Plastic limit } \\
(\%)\end{array}$ & $\begin{array}{c}\text { Plasticity index } \\
(\%)\end{array}$ \\
\hline \multicolumn{7}{|l|}{ Mud-volcano deposits: } \\
\hline 160-971D-3H-01, 130-150 & $\begin{array}{l}42.4 \\
40.3\end{array}$ & $\begin{array}{l}51.8 \\
26.4\end{array}$ & $\begin{array}{l}24.2 \\
26.4\end{array}$ & $\begin{array}{l}3.1 \\
60.2\end{array}$ & $\begin{array}{l}2.3 \\
18.2\end{array}$ & $\begin{array}{l}2.4 \\
42.1\end{array}$ \\
\hline $160-971 \mathrm{~B}-19 \mathrm{X}-01,130-150$ & 35.9 & 30.6 & - & 52.7 & 23.1 & 29.5 \\
\hline BAN-88 07-GC & 46.5 & - & - & 61 & 35.4 & 25.6 \\
\hline BAN-89 02-GC & 43.7 & - & - & 57.6 & 27.4 & 30.2 \\
\hline BAN-89 04-GC & 38.5 & - & - & 54.5 & 29.1 & 25.4 \\
\hline \multicolumn{7}{|l|}{ Transitional sediment: } \\
\hline $160-971 \mathrm{~A}-4 \mathrm{H}-02,132-150$ & 32.1 & 21.4 & 25.8 & 54.6 & 34.7 & 20 \\
\hline BAN-89 03-GC & 40.0 & & - & 53.7 & 26.7 & 27 \\
\hline \multicolumn{7}{|l|}{ Hemipelagic sediment: } \\
\hline
\end{tabular}

Table 6. Results from drained shear box tests on undisturbed samples and grain-size analyses from previous cruises.

\begin{tabular}{|c|c|c|c|c|c|c|c|c|c|}
\hline \multirow[b]{2}{*}{ Sample } & \multirow[b]{2}{*}{$\begin{array}{l}\text { Depth } \\
\text { (mbsf) }\end{array}$} & \multirow[b]{2}{*}{$\begin{array}{c}\text { Consolidation } \\
(\mathrm{kPa})\end{array}$} & \multicolumn{4}{|c|}{ Shear test } & \multicolumn{3}{|c|}{ Grain size analyses } \\
\hline & & & $\begin{array}{l}\text { Peak strength } \\
(\mathrm{kPa})\end{array}$ & $\begin{array}{l}\phi \mathrm{p} \\
\left({ }^{\circ}\right)\end{array}$ & $\begin{array}{l}\text { Residual strength } \\
\qquad(\mathrm{kPa})\end{array}$ & $\begin{array}{l}\phi \mathrm{r} \\
\left({ }^{\circ}\right)\end{array}$ & $\begin{array}{r}\text { Clay } \\
(\%)\end{array}$ & $\begin{array}{l}\text { Silt } \\
(\%)\end{array}$ & $\begin{array}{c}\text { Sand } \\
(\%)\end{array}$ \\
\hline \multicolumn{10}{|c|}{ Mud-volcano deposits } \\
\hline \multirow{2}{*}{ BAN-89 2-GC } & \multirow{2}{*}{$<5$} & 98.1 & 50.8 & \multirow{2}{*}{27.9} & 48.8 & \multirow{2}{*}{26.6} & \multirow{2}{*}{58} & \multirow{2}{*}{37} & \multirow{2}{*}{5} \\
\hline & & 198.1 & 104.1 & & 87.8 & & & & \\
\hline \multicolumn{2}{|c|}{ Transitional sediment } & & & & & & \multirow{3}{*}{52} & \multirow{3}{*}{38} & \multirow{3}{*}{10} \\
\hline \multirow[t]{2}{*}{ BAN-89 2-GC } & \multirow[t]{2}{*}{$<5$} & & & \multirow[t]{2}{*}{29.1} & & \multirow[t]{2}{*}{27.8} & & & \\
\hline & & 198.1 & 112.5 & & 107.0 & & & & \\
\hline
\end{tabular}

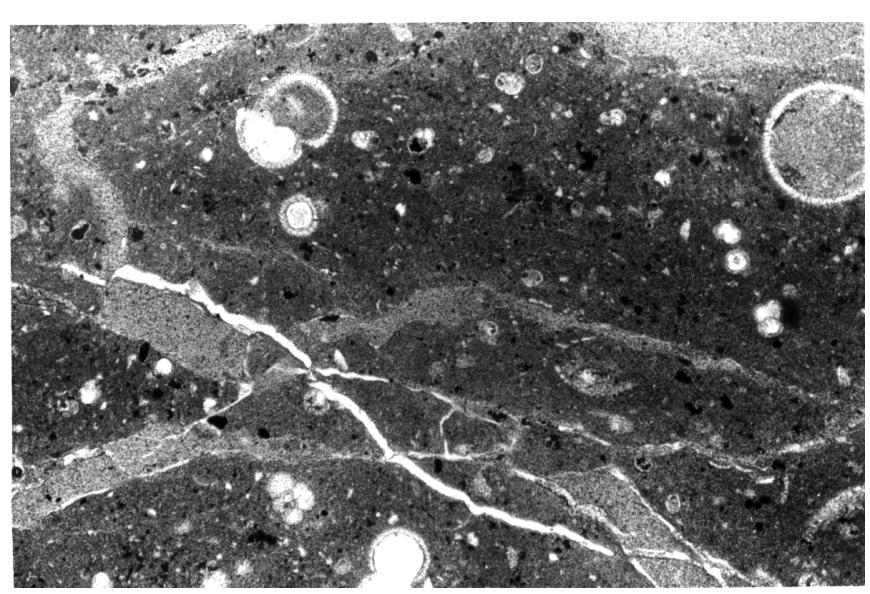

Figure 7. Microphotograph of sheared mud-volcano Sample 160-971B-16X03, 99-119 cm, with carbonaceous mud along the shear bands. Long axis is 2 $\mathrm{cm}$.

cannot be precisely identified (Fig. 8B). On vertical trims where a slight preconsolidation phase can be identified, the maximum precompression corresponds to effective stress of about $50 \mathrm{kPa}$. Slightly larger settlement is observed in samples trimmed horizontally. Hemipelagic oozes, for which only vertical and horizontal trims have been obtained, reveal a pre-consolidation phase up to effective stress of $1000 \mathrm{kPa}$. In contrast to the mud-volcano sediments, the largest settlement is obtained on the vertically trimmed specimen. The transitional sediment displays a consolidation curve of intermediate character between mud-volcano and hemipelagic sediments.

At 200 and $300 \mathrm{kPa}$ effective stress, the hydraulic conductivity in mud-volcano sediments is higher then in transitional sediments, although this sample was taken more than $100 \mathrm{~m}$ higher up in the succession (Fig. 8A; Table 2). At $500 \mathrm{kPa}$ and higher effective stress, when pre-consolidation stresses were presumably exceeded, mudvolcano deposits have similar or only slightly higher hydraulic conductivities than the transitional sample. At $700 \mathrm{kPa}$ effective stress, hydraulic conductivities of the different mud-volcano deposits drop drastically to values between 3.26 and $7.05 \times 10^{-9} \mathrm{~mm} / \mathrm{s}$, whereas the transitional sediment allows only $1.31-1.55 \times 10^{-8} \mathrm{~mm} / \mathrm{s}$ flux. This suggests that the apparent preconsolidation stress has been increased by the effects of "structure" (weak interparticle bonding), so that a higher stress than previously recorded is required for yielding to occur (Burland 1990; Lereouil and Vaughan, 1990). The permeabilities correlate well with results from grain-size analyzes, where the sample without a coarse component (i.e., sand) shows the minimum permeability (Sample 160-971B-16X-03, 99-119 cm).

In agreement with Olson and Daniel's (1981) observation, theoretical hydraulic conductivities calculated from oedometer tests on the Bannock specimens (Fig. 8B) are generally slightly higher than the hydraulic conductivities measured on undisturbed core samples (Table 2). For the maximum load of $2400 \mathrm{kPa}$ (not covered by direct measurements), hydraulic conductivities drop another order of magnitude, ranging between $7.8-9.1 \times 10^{-10} \mathrm{~mm} / \mathrm{s}$. The theoretical hydraulic conductivity of hemipelagic ooze (a crestal sample from Hilo dome; Sample BAN 89-08), is more than one order of magnitude higher than that of mud-volcano sediments. Values for the transitional sediment (Sample BAN 89-03; Table 2) lie somewhere between the hemipelagic ooze and the mud breccia. In Figure 7B, it is shown that the theoretical hydraulic conductivity of hemipelagic oozes is higher if calculated on the consolidation of a specimen trimmed horizontally, because of the preferential orientation of clay particles normally settled at the seafloor. The absolute value and the trend with increasing effective stress of hydraulic conductivity of mud-volcano sediments is instead nearly identical in vertical and horizontal trims, as well as in remolded specimens.

Previous studies show that the permeability of clays is sensitive to pore water salinities (e.g., Jose et al., 1989). Bearing in mind the extremely high values of salinity measured in the crestal region of the Napoli dome (i.e., up to $>300 \mathrm{~g} / \mathrm{kg}$; Emeis, Robertson, Richter, et al., 
A

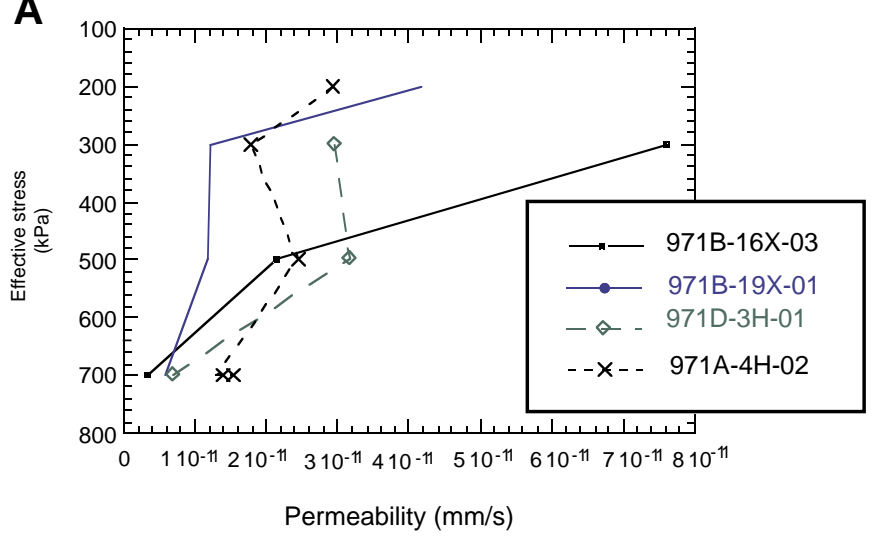

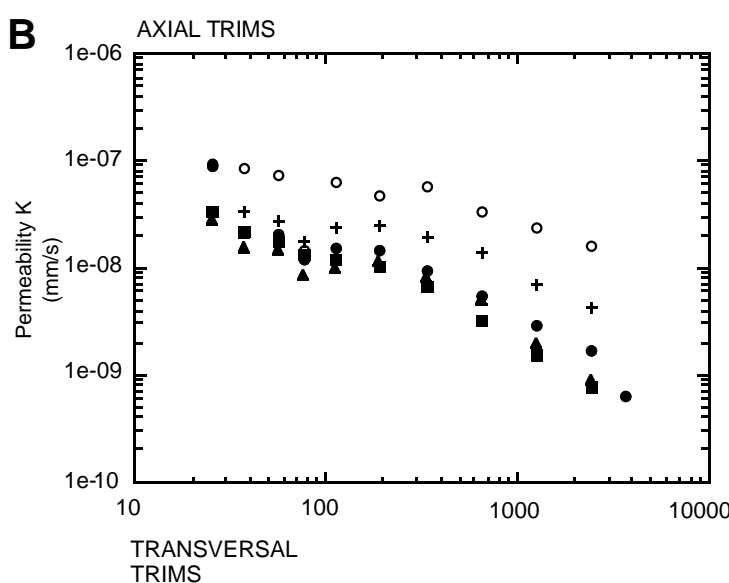
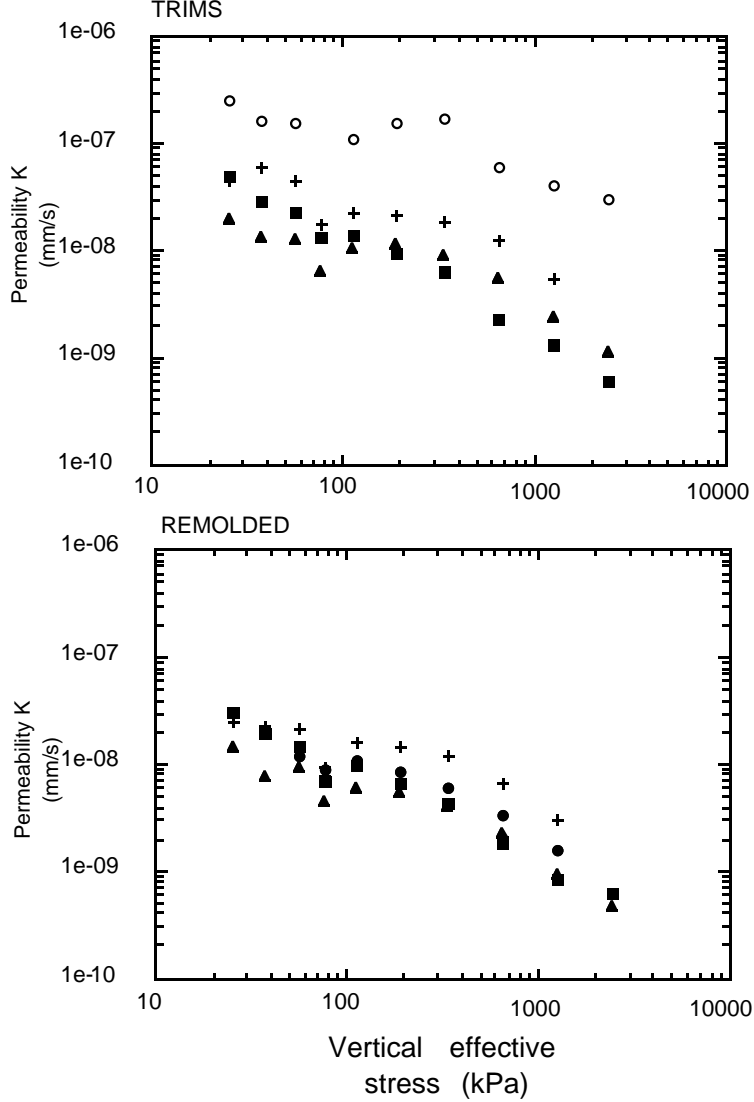

Figure 8. A. Results from permeability tests on whole-round samples from Leg 160, plotted as flow rate vs. effective stress $\left(\sigma^{\prime}\right)$. B. Theoretical permeability, $k$, determined from results of oedometer tests on Bannock samples of mud breccia (filled symbols), mixed sediment (crosses), and hemipelagic ooze (circles). Note the similarities between axial trims (top), transversal trims (middle), and remolded sediment (bottom). See text for discussion.

1996) we may expect our values to be higher than usual. Note also the presence of solid halite clasts in the crestal holes of Napoli dome and that halite precipitated after we split the core onboard the JOIDES Resolution as well as in the shear box (although the Leg 160 samples were remolded with tap de-aired, non-saline water). On the other hand, in the constant rate of flow tests, we used distilled water to avoid damaging the delicate transducers, which may have caused the opposite effect. Tap water underestimates the permeability because the clay minerals swell and thereafter might partly block the pores or porous disks in the permeability cell (e.g., Olson and Daniel, 1981). However, influx of only 1-3 mL per test was necessary to reach equilibrium in samples with a pore volume exceeding $100 \mathrm{ml}$. Moreover, the original pore fluid was partly expelled during initial consolidation, and remained in the tubes, and this was the first fluid to be pumped back into the samples; thereafter we alternated infusion and withdrawal of the pump to recycle the fluids. We therefore assume that we did not affect considerably the results of the direct permeability tests by not correctly matching permeant to the in situ pore fluid.

Temperature effects, as outlined previously (Anderson et al., 1977; Silva et al., 1989) can be ruled out for the tests, as (1) the procedures were carried out in an air-conditioned laboratory at $20^{\circ} \pm$ $0.5^{\circ} \mathrm{C}$, and (2) this temperature is close to the in situ temperatures where the samples were obtained, $\sim 16^{\circ} \mathrm{C}$ at the bottom of Hole $971 \mathrm{~B}$ (Emeis, Robertson, Richter, et al., 1996). Because of the lack of ma- 
terial, no permeability tests were undertaken on nannofossil ooze or sapropels. However, other permeability results by previous workers revealed that the sapropels are one hundred times more permeable than the predominantly clay- and silt-sized hemipelagic oozes and mud breccias (Schultheiss and Gunn, 1985; Westbrook, pers. comm., 1996). This might explain the thermal alteration (i.e., high vitrinite reflectance) of sapropel beds (Emeis, pers. comm., 1996) if migration of warm fluids can be predicted here (Westbrook et al., 1995).

\section{DISCUSSION}

\section{Shear Strength}

Regarding the results from shear tests, the dominance of minerals other than clays is unexpected (based on the internal angle of friction determined from shear tests; e.g., Kenney, 1967), whereas both XRD of the fine fraction (Robertson and Kopf, Chap. 45, this volume) and thin section study of mud-volcano deposits suggest kaolinite and smectite as the dominant mineral phases in the matrix. However, the concentration of calcareous mud along the shear surfaces in the deformed (initially perfectly homogenized) sapropel, and to a lesser extent in the mud breccia sample, suggest that some nannofossil ooze is present in all the sediments. If the amount of background sedimentation is variable, high friction angles from the Bannock as well as Leg 160 samples can be simply explained by variations in bulk mineralogy. Carbonate contents from both Bannock/Leg 160 samples are $23 \% / 21 \% \mathrm{CaCO}_{3}$ for mud breccia, $38 \% / 36 \% \mathrm{CaCO}_{3}$ for transitional sediment, and $65 \% / 53 \% \mathrm{CaCO}_{3}$ for hemipelagic ooze on average, and seem to control shear strength, plasticity and brittleness. Carbonate abundance is in good accordance with the friction angles determined from shear tests which indicate dominance of carbonaceous mud in the matrix (Arkin and Michaeli, 1989). Only one sample (Sample 160-971B-16X-03, 99-119 cm) shows friction angles in the clay mineral range (Tables 1, 6; e.g., Skempton, 1985). The results demonstrate that background sedimentation of nannofossil ooze is not restricted to intervals of inactivity of mud volcanoes, but takes place during phases of mud extrusion as well (see also Kopf et al., Chap. 49, this volume).

The plasticity index, often believed to reflect the abundance of clay and swelling mixed-layer clay minerals, mirrors the variation in clay and carbonate content. The Leg 160 sample recovered from the crest of Napoli dome (interval 160-971D-3H-01, 132-150 cm), consists of gaseous "mousse-like" mud, and has a high plasticity index (Fig. 4). The remaining mud-volcano deposits show smaller plasticity indices, and as carbonate contents increase (transitional and hemipelagic sediments), decreasing plasticity indices are obtained (Fig. 5 ). The amount of clay with its porous, mousse-like texture allows in situ fluid venting (Limonov et al., 1994; Cita et al., 1995), but changes dramatically if consolidated because of pore-space collapse (see below).

\section{Permeability and Consolidation Behavior}

Principally, permeabilities of hemipelagic sediments, determined both theoretically from oedometer tests and measured directly using a back-pressured apparatus, range in the same order of magnitude as other marine sediments (Schultheiss and Gunn, 1985). The permeability of mud-volcano deposits, however, range one to two orders of magnitude below that of clay-rich deep-sea clays from this extensive study by Schultheiss and Gunn (1985). The permeability results (Fig. 8) clearly illustrate that both differences in in situ compaction (i.e., the depth the sample was taken) as well as location of the holes (i.e., base, flank, crest) have no profound effects on the hydraulic conductivity. Grain-size variation, too, is fairly similar for the samples tested (Tables 1,6). However, the variable permeability of the different WR samples may have a secondary effect on processes like plugging vents or fault fissures and therefore fluid efflux (see below).
From the consolidation test we observe that within the range of applied effective stresses (0-2400 kPa) the mud-volcano sediments are more compressible than the host sediments, both in vertical and horizontal direction. However, the host sediments collapse in the higher effective stress applied $(>1000 \mathrm{kPa})$, where their compression index is higher than that of the mud-volcano sediments under the same load. This is because of the weak bondings developed among the carbonate particles of the oozes and to the more rigid skeleton formed by the biogenic components (Bryant et al., 1981). Also, the settlement during consolidation of the horizontally trimmed specimen is about $60 \%$ of the vertically trimmed specimen for nannofossil oozes, while the difference between the settlement of the both vertically and horizontally trimmed specimen of mud-volcano sediments is reduced to a few percent. This is an indication of preferred orientation of the solid particles in the oozes that does not exist in the mud breccia with its nearly homogeneous microfabric. The homogeneous structure of the solid skeleton of the mud-volcano deposits is also demonstrated by the similar behavior of theoretical hydraulic conductivity found in consolidation tests on vertical trims, horizontal trims, and remolded specimen (Fig. 8B). The trend of the coefficients of consolidation of the mud-volcano sediments vs. effective stress indicates that the sediment behaves as a remolded soil. Oozes display higher coefficient of consolidation than the mud-volcano sediments with a large difference between vertical and horizontal trims, thus suggesting in situ normal consolidation conditions. According to a standard geotechnical classification scheme, the mud-volcano sediments are saturated or slightly over-saturated, high-plasticity inorganic soils, with compression indices varying between 0.30 and 0.38 . The sediments show strong evidence of natural reworking prior to sampling.

The porosity of the mud-volcano sediments is generally lower (although highly variable) than that of the host nannofossil ooze (Fig. $4 \mathrm{~A}$ ). Consequently the wet density of the mud-volcano sediments is higher than that of the host sediments (Fig. 4B). This is in apparent contrast with the stratigraphic position of the mud-volcano sediments, which have moved upward to the sediment surface. The force of extrusion cannot be ascribed to a density difference with the surrounding sediments (diapiric mode), at least near the surface. Additional driving forces must be in the presence of gaseous phases and overpressured fluids (e.g., Yassir and Bell, 1994). The presence of gas in the mud breccia has been demonstrated on cores by geochemical analyses (Emeis, Robertson, Richter, et al., 1996) and is expected to cause undersaturation in the laboratory specimen. The apparent saturation and oversaturation found in our samples could be explained by the patchy distribution of the gas (e.g., derived by dissociation of gas hydrate crystals). The presence of overpressured fluid in the liquid phase is supported by the observed saturation of our samples. These fluids could also contain a minor fraction of dissolved gas pervasively distributed in the pore space as a result of the high in situ confining pressures, but then should have transformed into free gas after sampling and thus result in undersaturation. We conclude that pervasive gas, if present, had such low concentrations that the mechanical properties of the samples were not affected.

The nearly isotropic behavior of the mud breccia to consolidation and water flow through the pore space (Fig. 8B) suggest that these sediments hardly preserve fabrics from their ascent or subsequent settling. This observation is best explained having occurred under zero effective stress conditions and thus when the mud was completely liquefied during formation of a sedimentary diatreme according to the model of Brown (1990). The mud must have generated fast, most likely as low-viscosity debris flows. The presence of clasts up to 50 $\mathrm{cm}$ across in the debris -flow deposits requires either high flow velocities or high viscosities, the matter of which can be excluded. 


\section{Implications for Mechanisms of Mud-Volcano Emplacement}

Reconstruction of mud-volcano evolution provides evidence of interfingering of stratified mud debris flows and hemipelagites, as well as a trend from coarse polymictic gravels to matrix-supported muds of variable clast contents, and subsequently mousse-like clayey silts as the youngest mud volcano. This variation in mud-volcano deposits must record the history of the sourcing and transport mechanisms (Robertson et al., 1996). The depth of origin of the mud and clasts remains in question and each must be treated separately, as there is no clear evidence suggesting that they were derived together. It has been assumed that the mud is trapped somewhere in or beneath the prism, resisting consolidation because of overpressuring.

The occurrence of mud domes on the accretionary prism was related to backthrust faults in the inner part of the Mediterranean Ridge accretionary complex (Fig. 9; Camerlenghi et al., 1995). Initiation of mud volcanism was possibly contingent on puncturing a seal of Messinian evaporite or accreted strata, which allowed clast-bearing muds to rise and be extruded onto the seafloor as multiple debris flows. Propagation or reactivation of high-angle reverse faults that intersect both the salt layers and accreted strata is postulated to break the seal and allow unlithified material to extrude. This mechanism requires pore fluid pressures in the escaping mud to be at or near lithostatic values to overcome the fault-normal closure stress. This crite- rion can be relaxed to some extent if favorably oriented wrench faults are present (Fig. 9; Barber et al., 1986; Reed et al., 1990; Vernette et al., 1992). Alternatively, the pressure may rise to values sufficient to fracture the rock (as described for accretionary wedges; e.g., Behrmann, 1991), so that the diapirs are actively emplaced rather than merely being permitted to rise under buoyancy. By studying thin sections of mudstone clasts from these sediments, we found indications of hydrofracture (Robertson and Kopf, Chap. 45, this volume).

The overpressure in the source clay formation must be predominantly primary in nature (i.e., caused by delayed consolidation) and not acquired later, since the extruded mud is still plastic after millions of years of burial. Realistically, this requires that the source layer is relatively thick and of low permeability. The latter constraint, which we have verified experimentally, will also enhance secondary contributors to overpressuring that we believe to be likely in this setting: partial dehydration of the smectitic clays and thermogenic generation of gases. As with mud-volcano fields elsewhere in the world, the extra pressure charge caused by methane and further gas expansion during upwards movement of the diapiric mud is probably responsible for the domes displaying effusive mud volcanicity at the seafloor. Variable gas and fluid supply during formation, presumably from some depth, is believed to be the main controlling parameter on the timing of mud volcanic activity.

Studies of thermal maturity of organic compounds within the mud-volcano deposits imply a Messinian age of the mud that has then

\section{Northeast}

\section{Hellenic trench (backstop, empty fore-arc basin)}

\section{Aegean Sea} (back-arc basin)

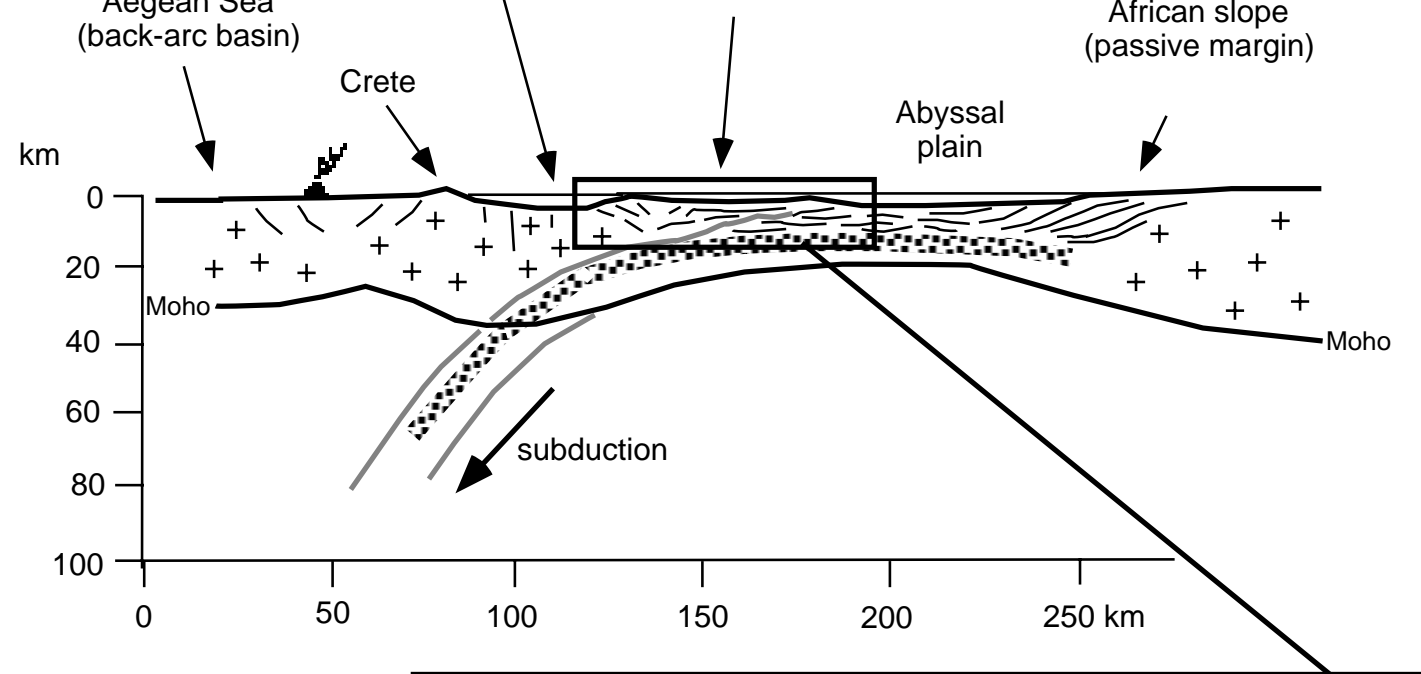

\section{Southwest}

Mediterranean Ridge accretionary complex

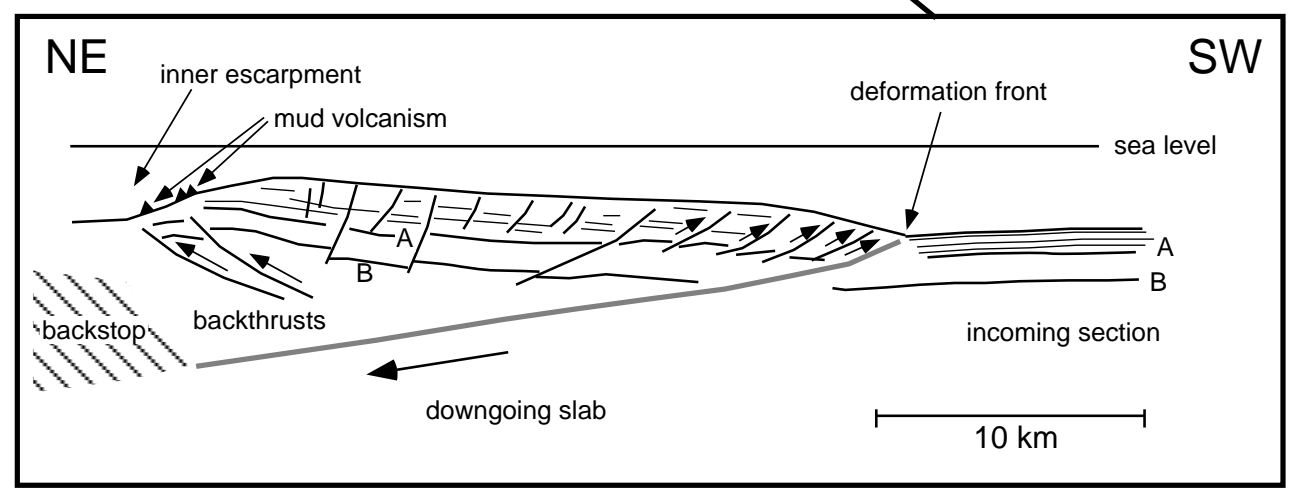

Figure 9. Interpreted cross sections showing the tectonic setting of the mud volcanoes at the inner escarpment of the Mediterranean Ridge accretionary complex. The line labelled A illustrates the presumed level of the base of the Pliocene-Quaternary sediments, and the line labelled B is the interpreted base of the Messinian evaporites (according to Chaumillon and Mascle, 1995). For location see Figure 1. 
undergone subduction along the décollement before it was allowed to remigrate upward along backthrust faults (Schulz et al., 1997). As demonstrated in the case of the Barbados (e.g., Martin et al., 1996) and postulated for the Nankai subduction systems, gas, predominantly methane, follows the subduction trace. This implies that high-pressure fluids derived deep within the subduction zone control the variation in mud-volcano activity by variable mobilization and pulsed upward-migration (Yassir and Bell, 1994).

\section{ACKNOWLEDGMENTS}

The authors thank Najwa Yassir and Al Bolton for their detailed suggestions which greatly improved the manuscript. Alastair Lumsden is acknowledged for use of the laboratory and geotechnical equipment in Earth Sciences, University of Leeds, as is Doug Stewart for loan of the hydrostatic permeability cell. Graham Westbrook is thanked for discussion and providing flow pumps and instrumentation for permeability tests. Funding for this research was received through DFG Grant Be-1041/10 and NERC Grant GST 02/835.

\section{REFERENCES}

Agar, S.M., 1990. The interaction of fluid processes and progressive deformation during shallow level accretion: examples from the Shimanto Belt of SW Japan. J. Geophys. Res., 95:9133-9148.

Anderson, R.N., Langseth, M.G., and Sclater, J.G., 1977. The mechanisms of heat transfer through the floor of the Indian Ocean. J. Geophys. Res., 82:3391-3409.

Arkin, Y., and Michaeli, L., 1989. Strength and consistency of artificial claycarbonate mixtures: simulation of natural sediments. Eng. Geol., 26:201213

ASTM, 1990. Standard test method for measurement of hydraulic conductivity of saturated porous materials using a flexible wall permeameter. Philadelphia (Am. Soc. Testing and Mater.), D5084.

_ 1991. Standard test method for direct shear tests of soils under consolidated drained conditions. Philadelphia (Am. Soc. Testing and Mater.), D3080-90.

Barber, A.J., Tjokrosapoetro, S., and Charlton, T.R., 1986. Mud volcanoes, shale diapirs, wrench faults, and melanges in accretionary complexes, Eastern Indonesia. AAPG Bull., 70:1729-1741.

Behrmann, J.H., 1991. Conditions for hydrofracture and the fluid permeability of accretionary wedges. Earth Planet. Sci. Lett., 107:550-558.

Bjerrum, L., 1954. Geotechnical properties of Norwegian marine clays. Geotechnique, 4:49-69.

Borradaile, G.J., 1981. Particulate flow and the formation of cleavage. Tectonophysics, 72:305-321.

Boyce, R.E., 1976. Definitions and laboratory techniques of compressional sound velocity parameters and wet-water content, wet-bulk density, and porosity parameters by gravimetric and gamma-ray attenuation techniques. In Schlanger, S.O., Jackson, E.D., et al., Init. Repts. DSDP, 33: Washington (U.S. Govt. Printing Office), 931-958.

Breen, N.A., Silver, E.A., and Hussong, D.A., 1986. Structural styles of an accretionary wedge south of the island of Sumba, Indonesia, revealed by SEAMARC II side scan sonar. Geol. Soc. Am. Bull., 97:1250-1261.

Brown, K.M., 1990. The nature and hydrogeologic significance of mud diapirs and diatremes for accretionary systems. J. Geophys. Res., 95:89698982.

Brown, K.M., and Westbrook, G.K., 1988. Mud diapirism and subcretion in the Barbados Ridge accretionary complex: the role of fluids in accretionary processes. Tectonics, 7:613-640.

Bryant, W.R., Bennett, R.H., and Katherman, C.E., 1981. Shear strength, consolidation, porosity, and permeability of oceanic sediments. In Emiliani, C. (Ed.), The Sea (Vol. 7): The Oceanic Lithosphere: New York (Wiley), 1555-1616.

Bryant, W.R., Hottman, W., and Trabant, P., 1975. Permiability of unconsolidated and consolidated marine sediments, Gulf of Mexico. Mar. Geotechnol., 1:1-14.
BS 1377, 1991. Methods of test for soils for civil engineering purposes. Brit. Stand. Inst.

Burland, J.B., 1990. On the compressibility and shear strength of natural clays. Geotechnique, 40:329-378.

Camerlenghi, A., 1991. Il diapirismo d'argilla sulla Dorsale Mediterranea [Ph.D. thesis]. Univ. of Milano.

Camerlenghi, A., Cita, M.B., Della Vedova, B., Fusi, N., Mirabile, L., and Pellis, G., 1995. Geophysical evidence of mud diapirism on the Mediterranean Ridge accretionary complex. Mar. Geophys. Res., 17:115-141.

Camerlenghi, A., Cita, M.B., Hieke, W., and Ricchiuto, T.S., 1992. Geological evidence for mud diapirism on the Mediterranean Ridge accretionary complex. Earth Planet. Sci. Lett., 109:493-504.

Casagrane, A., 1936. The determination of preconsolidation load and its practical significance. In Casagrande, A., Rutledge, P.C., and Watson, J.D. (Eds.), Proc. 1st Int. Conf. Soil Mech. Found. Eng., Am. Soc. Civ. Eng., 3:60-64.

Casagrande, A., 1948. Classification and identification of soils. Trans. Am. Soc. Civ. Eng., 113:901-992.

Chaumillon, E., and Mascle, J., 1995. Variation laterale des fonts de deformation de la Ride mediterraneenne (Mediterranee orientale). Bull. Soc. Geol. Fr. 166:463-478.

Cita, M.B., and Camerlenghi, A., 1990. The Mediterranean Ridge as an accretionary prism in collision context. Mem. Soc. Geol. Ital., 45:463480.

Cita, M.B., Camerlenghi, A., Erba, E., McCoy, F.W., Castradori, D., Cazzani, A., Guasti, G., Giambastiani, M., Lucchi, R., Nolli, V., Pezzi, G., Redaelli, M., Rizzi, E., Torricelli, S., and Violanti, D., 1989. Discovery of mud diapirism in the Mediterranean Ridge: a preliminary report. Boll. Soc. Geol. Ital., 108:537-543.

Cita, M.B., Woodside, J.M., Ivanov, M.K., Kidd., R.B., and Limonov, A.F., 1995. Fluid venting from a mud volcano in the Mediterranean Ridge diapiric belt. Terra Nova, 7:453-458.

Clennell, M.B., 1992. The mélanges of Sabah, Malaysia [Ph.D. thesis]. Univ. London.

Cronin, B.T., Ivanov, M.K., Limonov, A.F., Egorov, A., Akhmanov, G.G., Akhmetjanov, A.M., Kozlova, E.V., and Shipboard Scientific Party TTR3, 1997. New discoveries of mud volcanoes on the Eastern Mediterranean Ridge. J. Geol. Soc. London, 154:173-182.

Demars, K.R., and Chaney, R.C. (Eds.), 1992. Geotechnical properties, behaviour and performance of calcareous soils. Am. Soc. for Testing and Mater., ASTM STP 777.

Emeis, K.-C., Robertson, A.H.F., Richter, C., et al., 1996. Proc. ODP, Init. Repts., 160: College Station, TX (Ocean Drilling Program).

Flecker, R., and Kopf, A., 1996. Data report: clast and grain size analysis of sediment recovered from the Napoli and Milano mud volcanoes, Eastern Mediterranean. In Emeis, K.-C., Robertson, A.H.F., Richter, C., et al., Proc. ODP, Init. Repts., 160: College Station, TX (Ocean Drilling Program), 529-532.

Fowler, S.R., White, R.S., and Louden, K.E., 1985. Sediment dewatering in the Makran accretionary prism. Earth Planet. Sci. Lett., 75:427-438.

Head, K.H., 1980. Manual of Soil Laboratory Testing (Vol. 1): Soil Classification and Compaction Tests (1st ed.): New York (Halsted Press/Wiley).

Henry, P., Le Pichon, X., Lallemant, S., Foucher, J.-P., Westbrook, G., and Hobart, M., 1990. Mud volcano field seaward of the Barbados accretionary complex: a deep-towed side scan sonar survey. J. Geophys. Res., 95:8917-8929.

Jose, U.V., Bhat, S.T., and Nayak, B.U., 1989. Influence of salinity on permeability characteristics of marine sediments. Geotechnology, 8:249258.

Kenney, T.C., 1967. The influence of mineralogical composition on the residual strength of natural soils. Proc. Geotech. Conf. Shear Properties of Soils and Rocks, Oslo, 1:123-129.

Knipe, R.J., 1986. Deformation mechanism paths for sediments undergoing lithfication. Mem.?Geol. Soc. Am., 166:151-160.

Kopf, A., Robertson, A.H.F., Clennell, M.B., and Flecker, R., in press. Mechanisms of mud extrusion on the Mediterranean Ridge Accretionary Prism. Geo-Mar Lett.

Kopf, A., Sample, J.C., Bauer, P., Behrmann, J.H., and Erlenkeuser, H., 1995. Diagenetic carbonates from Cascadia Margin: textures, chemical compositions, and oxygen and carbon stable isotope signatures. In Carson, B., Westbrook, G.K., Musgrave, R.J., and Suess, E. (Eds.), Proc. ODP, Sci. 
Results, 146 (Pt 1): College Station, TX (Ocean Drilling Program), 117136.

Lambe, T.W., and Whitman, R.V., 1969. Soil Mechanics: New York (Wiley).

Lee, H.J., 1985. State of the art: laboratory determination of the strength of marine soils. In Chaney, R.C., and Demars, K.R. (Eds.), Strength Testing of Marine Sediments: Laboratory and In-Situ Measurements: ASTM Spec. Tech. Publ., 883:181-250.

Lerouiel, S., and Vaughan, P.R., 1990. The general and congruent effects of structure in natural soils and rocks. Geotechnique, 40:467-488.

Limonov, A.F., Woodside, J.M., and Ivanov, M.K. (Eds.), 1994. Mud Volcanism in the Mediterranean and Black Seas and Shallow Structure of the Eratosthenes Seamount. Initial Results of the Geological and Geophysical Investigations during the Third "Training-through-Research" Cruise of the R/V Gelendzhik (June-July 1993). UNESCO Rep. Mar. Sci., 64.

Lupini, J.F., Skinner, A.E., and Vaughan, P.R., 1981. The drained residual strength of cohesive soils. Geotechnique, 31:181-213.

Maltman, A. (Ed.), 1994. The Geological Deformation of Sediments: London (Chapman \& Hall)

Martin, B.M., Kastner, M., Henry, P., Le Pichon, X., and Lallement, S., 1996. Chemical and isotopic evidence for sources of fluids in a mud volcano field seaward of the Barbados accretionary wedge. J. Geophys. Res., 101:20325-20345.

Moore, R., 1991. The chemical and mineralogical controls upon the residual strength of pure and natural clays. Geotechnique, 41:35-47.

Olson, R.E., and Daniel, D.E., 1981. Measurement of the hydraulic conductivity of fine-grained soils. In Zimmie, T.F., and Riggs, C.O. (Eds.), Permeability and Groundwater Contaminant Transport. ASTM Spec. Tech. Publ., 746:18-64.

Reed, D.L., Silver, E.A., Tagudin, J.E., Shipley, T.H., and Vrolijk, P., 1990. Relations between mud volcanoes, thrust deformation, slope sedimentation and gas hydrates, offshore north Panama. Mar. Pet. Geol., 7:44-54.

Robertson, A., and Ocean Drilling Program Leg 160 Scientific Party, 1996. Mud volcanism on the Mediterranean Ridge: Initial results of Ocean Drilling Program Leg 160. Geology, 24:239-242.

Schultheiss, P.J., and Gunn, D.E., 1985. The permeability and consolidation of deep-sea sediments. Inst. Oceanogr. Sci. Rep., 201.

Schulz, H.M., Emeis, K.C., and Volkmann, N., 1997. Organic carbon provenance and maturity in the mud breccia from the Napoli mud volcano: indicators of origin and burial depth. Earth Planet. Sci. Lett., 144:141151.
Shipley, T.H., Stoffa, P.L., and Dean, D.F., 1990. Underthrust sediments, fluid migration paths, and mud volcanoes associated with the accretionary wedge off Costa Rica: Middle America trench. J. Geophys. Res., 95:8743-8752.

Silva, A.J., Hetherman, J.R., and Calnan, D.I., 1989. Low-gradient permeability testing of fine-grained marine sediments. In Zimmie, T.F., and Riggs, C.O. (Eds.), Permeability and Groundwater Contaminant Transport. ASTM STP, 746:121-136.

Skempton, A.W., 1964. The long term stability of clay slopes. Geotechnique, 14:77.

Skempton, J.A.W., 1985. Residual strength of clays in landslides, folded strata and the laboratory. Geotechnique, 35:3-18.

Tavenas, F., Leblond, P., Jean, P., and Leroueil, S., 1983a. The permeability of natural soft clays. Part 1: Methods of labororatory measurement. Can. Geotech. J., 20:629-644.

, $1983 \mathrm{~b}$. The permeability of natural soft clays. Part 2: Permeability characteristics. Can. Geotech. J., 20:645-660.

Taylor, E., and Leonard, J., 1990. Sediment consolidation and permeability at the Barbados forearc. In Moore, J.C., Mascle, A., et al., Proc. ODP, Sci. Results, 110: College Station, TX (Ocean Drilling Program), 289-308.

Vernette, G., Mauffret, A., Bobier, C., Briceno, L., and Gayet, J., 1992. Mud diapirism, fan sedimentation and strike-slip faulting, Caribbean Colombian Margin. Tectonophysics, 202:335-349.

Westbrook, G.K., and MEDRIFF Consortium, 1995. Three brine lakes discovered in the seafloor of the Eastern Mediterranean. Eos, 76/33:313315.

Yassir, N., 1990. The undrained shear behavior of fine sediments. In Knipe, R.J., and Rutter, E.H. (Eds.), Deformation Mechanisms, Rheology and Tectonics. Geol. Soc. Spec. Publ. London, 54:399-404.

Yassir, N.A., and Bell, J.S., 1994. Relationships between pore pressures, stresses and present-day geodynamics in the Scotian Shelf, Offshore Eastern Canada. AAPG Bull., 78:1863-1880.

Date of initial receipt: 27 January 1997

Date of acceptance: 22 June 1997

Ms 160SR-049 